\title{
1 beditor: A computational workflow for designing libraries of guide RNAs for CRISPR-mediated base editing
}

3 Rohan Dandage ${ }^{\star 1,2,3,4}$, Philippe C Després ${ }^{1,2,3}$, Nozomu Yachie ${ }^{5,6,7,8}$, Christian R 4 Landry ${ }^{1,2,3,4}$

6 1. Département de Biochimie, Microbiologie et Bio-informatique, Faculté de sciences et 7 genie, Université Laval, Québec, Québec, G1V 0A6, Canada

8 2. PROTEO, The Québec Research Network on Protein Function, Structure and 9 Engineering, Université Laval, Québec, Québec, G1V 0A6, Canada

10 3. Centre de Recherche en Données Massives (CRDM), Université Laval, Québec, 11 Québec, G1V 0A6, Canada

12 4. Département de Biologie, Faculté de sciences et Génie, Université Laval, Québec, 13 Québec, G1V 0A6, Canada

14 5. Research Center for Advanced Science and Technology, University of Tokyo, 4-6-1 15 Komaba, Meguro-ku, Tokyo 153-8904, Japan.

16 6. Department of Biological Sciences, Graduate School of Science, University of Tokyo, 17 7-3-1 Hongo, Bunkyo-ku, Tokyo 113-0033, Japan

18 7. Institute for Advanced Biosciences, Keio University, 14-1 Baba-cho, Tsuruoka, 19 Yamagata 997-0035, Japan

20 8. Graduate School of Media and Governance, Keio University, 5322 Endo, Fujisawa, 21 Kanagawa 252-0882, Japan

$22{ }^{*}$ To whom correspondence should be addressed. Email: rohan.dandage.1@bio.ulaval.ca 


\section{ABSTRACT}

2 CRISPR-mediated base editors have opened unique avenues for scar-free genome-wide 3 mutagenesis. Here, we describe a comprehensive computational workflow called beditor 4 that can be broadly adapted for designing guide RNA libraries with a range of CRISPR5 mediated base editors, PAM recognition sequences and genomes of many species. 6 Additionally, in order to assist users in selecting the best sets of guide RNAs for their 7 experiments, a priori estimates, called beditor scores are calculated. These beditor scores 8 are intended to select guide RNAs that conform to requirements for optimal base editing: 9 the editable base falls within maximum activity window of the CRISPR-mediated base 10 editor and produces non-confounding mutational effects with minimal predicted off-target 11 effects. We demonstrate the utility of the software by designing guide RNAs for base12 editing to create or remove thousands of clinically important human disease mutations. 


\section{INTRODUCTION}

CRISPR-mediated base editors (BEs) are engineered by fusing a DNA modifying protein with a nuclease-defective Cas9 (dCas9) protein, allowing scar-free targeted mutagenesis $(1-5)$. Currently, two major types of BEs are available - cytosine base editors (CBEs) that enable the conversion of cytosine to uracil by catalysis and then to thymine through replication or repair, $(1,6-8)$-for example BE3 (6) and Target-AID (C.G to T•A) (1). Similarly adenine base editors (ABEs) enable conversion of adenine to inosine by catalysis and then to guanine through replication or repair $(A \cdot T$ to $G \cdot C)(2,9,10)$-for example ABE7.10 (2). Currently BEs enable many codon level substitutions and thus amino acid substitutions (Figure S1). Owing to the unique capability of scar-free mutagenesis, BEs have recently found numerous applications in both model and nonmodel organisms $(3,10-13)$ and have substantial promise in therapeutic applications (14,

13 15).

14 For designing guide RNAs (gRNA) in BE mediated mutagenesis experiments, several specific requirements for the optimal activities of DNA modifying and Protospacer Adjacent Motif (PAM) sequences need to be taken into consideration. The complexity of this task especially increases when designing gRNA libraries against large sets of targets located across a given genome. Because design of gRNA sequences is a primary requirement for the success of the BE-mediated mutagenesis experiment, the methods and strategies involved in designing gRNA libraries are arguably the one of the most important factors in such experiments.

Currently available gRNA designing tools are however either specifically focused on nonsense mutations

or allow very limited customization (BioRxiv: 24 https://doi.org/10.1101/373944) (See Table S1), leaving a major roadblock in the applications of the BEs in genome editing. The continuous discoveries of new BEs and the expansion of their existing editing capabilities (17) demand a complementary development in computational methods that would allow designing gRNAs using new and improved base editors. Moreover, considering the prospective applications across nonmodel organisms, compatibility with diverse genomes is essential. Overall, therefore, a comprehensive computational framework to design gRNAs libraries can potentially fuel the progress in CRISPR-base editing technology and its diverse applications in genome 32 editing.

We developed a comprehensive computational workflow called beditor (Figure 1a) that can design gRNA libraries with any requirements of DNA modifying enzyme. These include the range of nucleotides where maximum catalytic activity of BE occurs, henceforth simply referred to as 'activity window', and PAM recognition sequence. beditor 38 database (18) and any annotated custom genomes. Additionally, the beditor workflow 
1 also provides a priori estimates called beditor scores for each gRNA that accounts for 2 specific editing requirements of BEs, gRNA binding at off-target sites and the number and 3 types of off-target effects (Figure 1b). Such estimates will inform researchers in optimizing 4 throughput of their BE-mediated mutagenesis experiments. With its Graphical User 5 Interface (GUI), command line interface and open source Application programming 6 interface (API), the beditor workflow has broad applicability for the genome editing 7 community and its open source implementation will allow for continuous enhancements 8 in the future.

\section{MATERIALS AND METHODS}

\section{Implementation}

12 The beditor workflow is implemented as an open-source python 3.6 package hosted at $13 \mathrm{https}: / /$ pypi.org/project/beditor. The source code of beditor can be accessed at $14 \mathrm{https}: / / \mathrm{www}$.github.com/rraadd88/beditor. The documentation of the software with API is 15 available at https://www.github.com/rraadd88/beditor/README.md. The beditor workflow 16 depends on other open source softwares such as PyEnsembl (19), BEDTools(20), 17 BWA(21) and SAMtools(22) at various steps of the analysis. User provided mutation 18 information is first checked for validity with PyEnsembl 19 (https://github.com/openvax/pyensembl.). Genomic sequences flanking the mutation 20 sites are fetched using BEDTools (20). The designed gRNAs are aligned the reference 21 genome using BWA (21) and alignments are processed using SAMtools (22) for 22 evaluation of off-target effects using the beditor scoring system. Visualization of 23 alignments of guide RNAs with genomic DNA are created using the DnaFeaturesViewer 24 package (https://github.com/Edinburgh-Genome-Foundry/DnaFeaturesViewer).

\section{5 beditor scoring system}

26 Alignment of the designed gRNAs (with PAM sequence) with the provided reference 27 genome is carried out using BWA, as used in (21), allowing for a maximum of two 28 mismatches per alignment (23). The beditor score is evaluated as follows.

$$
\begin{gathered}
P_{i}=\left\{\begin{array}{cc}
P_{\min } & \text { if } \text { mismatch is near PAM } \\
: & : \\
P_{\max } & \text { if } \text { mismatch distant from PAM }
\end{array}\right. \\
P_{a}=\prod_{i=1}^{M_{\max }} P_{i}
\end{gathered}
$$




$$
\begin{aligned}
G_{a} & = \begin{cases}G_{g} & \text { if genic } \\
G_{i g} & \text { if intergenic }\end{cases} \\
B & =\left(\prod_{a=1}^{n} P_{a} * G_{a}\right) * A
\end{aligned}
$$

1 For an alignment between a gRNA sequence and the genome, $P_{i}$ is a penalty assigned 2 to a nucleotide in the gRNA sequence based on the position of a mismatch in the aligned 3 sequence relative to the PAM. If the mismatch is near the PAM sequence, a minimal 4 penalty $P_{\min }$ is assigned. Conversely, if the mismatch is far from the PAM, a maximum 5 penalty $P_{\max }$ is assigned. The relative values of such penalties were determined by fitting 6 a third degree polynomial equation to the mismatch tolerance data from (24) (Figure S2). 7 This way, penalties increase non-linearly from $P_{\min }$ to $P_{\max }$, as the distance of nucleotide 8 (i) from PAM sequence increases. Individual penalties assigned for all the nucleotides in 9 a gRNA are then multiplied to estimate a penalty score for a given alignment called $P_{a}$ 10 (equation 2). In cases of gRNAs with lengths other than 20, the fitted equation is used to 11 interpolate penalty scores. In case of 5 ' PAMs, the order of the vector containing position 12 wise penalty scores is reversed. $G_{a}$ is a penalty defined by whether the off-target 13 alignment lies within a genic or an intergenic region (equation 3 ). $A$ is a penalty based on 14 whether the editable base lies within the activity window of BE (equation 4).

15 Note that due to the lack of large scale BE editing data, in the current version of beditor, 16 penalties are set based on the importance of each requirement (Table S2). Such penalties 17 would be informed from empirical data in the future developments.

18 The overall beditor score $B$ for a gRNA is determined by multiplying penalties assigned 19 per alignment $\left(P_{a}\right.$ and $\left.G_{a}\right)$ for all alignments $(n)$ with a penalty assigned to the gRNA (A) 20 (equation 4). Multiplication of individual penalties insures that if any of the criteria is 21 suboptimal, the beditor score decreases.

\section{Demonstrative analysis 1: customizability with respect to base editing strategies}

23 For a demonstrative analysis with custom base editors and PAM recognition sequences 24 were used (Figure 2 and Table S3), 1000 nucleotide and amino acid mutations were 25 randomly assigned across the genome of $S$. cerevisiae 26 (https://github.com/rraadd88/test_beditor). Such sets of mutations create uniform 27 datasets ideal for testing features of beditor. The input mutation data was created for both 28 mutation formats (either amino acid or nucleotide) and modes of mutagenesis ("create" 29 or "remove"). . The command "beditor --cfg params.yml" was executed. Here, params.yml 30 contains input parameters of the analysis (Table S4) in a user-friendly YAML format. For 31 this analysis, input parameter 'host' was set to 'Saccharomyces_cerevisiae' and 
1 parameter 'genomeassembly' was set to 'R64-1-1'. Summary statistics on the editability

2 are included as Table S5 and gRNA libraries are included as Supplementary data 1.

\section{Demonstrative analysis 2: ability to work with different species}

4 For a demonstrative analysis with different species, sets of 1000 random mutations were 5 created in genome of 10 representative species (Bos Taurus, Danio rerio, Equus 6 caballus, Felis catus, Gallus gallus, Macaca fascicularis, Mus musculus, Pan paniscus, 7 Saccharomyces cerevisiae and Sus scrofa) as described in case of demonstrative 8 analysis 1 . The input parameters used in this analysis are the same as Table S4 except 9 for host names and genome assembly versions that were obtained from $10 \mathrm{http}: / /$ useast.ensembl.org/index.html. The summary statistics on the editability are 11 included as Table S6 and gRNA libraries are included as Supplementary data 2.

\section{Case study analysis}

14 For the case study analysis, a set of clinically associated human mutations were obtained 15 from the Ensembl database in GVF format (ftp://ftp.ensembl.org/pub/release16 93/variation/gvf/homo_sapiens/homo_sapiens_clinically_associated.gvf.gz, Date 17 modified: 08/06/2018, 16:13:00). From genomic coordinates of SNPs, inputs for 18 nucleotide mutations (reference and mutated nucleotide) and amino acid mutations 19 (transcript ids, amino acid position, reference residue and mutated residue) were 20 identified using PyEnsembl (19). The mutation information was provided to the beditor 21 workflow as a tab-separated file. The input parameters used in this analysis are the same 22 as Table S4 except for the host name - 'homo_sapiens' and genome assembly version23 'GrCh38'. Summary statistics on the editability are included as Table S7 and gRNA 24 libraries are included as Supplementary data 3. 


\section{RESULTS}

\section{Design of beditor workflow}

3 The beditor workflow contains sequential steps that lead from input target sequences and 4 other input parameters to the designed gRNA libraries as output (Figure 1a). The user provides information about the desired set of mutations as an input and a library of gRNAs is generated with the corresponding a priori estimates called beditor scores to help users in selecting the best performing gRNAs. beditor can also be used to execute only a subset of the analysis steps by changing the input parameters or providing inputs for intermediate steps. The standard input of beditor depends on the format of mutations i.e. nucleotide or amino acid. To carry out nucleotide level mutations, the users need to provide genome coordinates and the desired nucleotide after mutagenesis. For carrying out amino acid level mutations, the users provide Ensembl stable transcript ids, the position of the targeted residues and the corresponding mutated residue. Users can also provide inputs to limit the amino acid substitutions to a custom substitution matrix and specify whether only non-synonymous or synonymous substitutions should be carried out. In addition to creating mutations on a wild-type background ('create' mode), the beditor workflow also provides an option to design guides that would remove alternative SNPs and to mutate to the reference or wild-type alleles ('remove' mode). The program can be accessed via GUI (Figure S3), command line or API. In addition to the gRNAs designed to carry out provided mutations, the beditor workflow can also design control gRNAs that are important in the large-scale mutagenesis experiments. The positive control gRNAs designed by beditor generate non-sense mutations in the coding region of interest and negative control gRNAs that lack an editable nucleotide in the editing window of the BE,

24 thus expected to have null effect.

\section{Customizability for broad utility}

26 The beditor workflow utilizes a PyEnsembl python API (19) to fetch and work with the genomes of over 125 species and their various assemblies from the Ensembl genome database $(18,25)$, providing a broad utility for researchers across a wide spectrum of fields. beditor is also compatible with any custom user-made annotated genome. The ability to carry out parallel processing allows for the design of large gRNA sequence libraries using minimal computational resources (Figure S4). The users can incorporate BEs with varied editing properties and even novel BEs as per requirements. Similarly, they can incorporate any custom PAM sequences (for example Table S3) in addition to the experimentally validated PAMs already incorporated in the current version of beditor (listed in Table S8). Additionally, the location of the PAM with respect to the gRNA (upstream or downstream) and the provided length of gRNA are taken into consideration

37 in the analysis. Lastly, beditor scores allows the users to select the best set of gRNAs for 38 mutagenesis experiments. 


\section{Selecting the best performing gRNAs}

We defined a novel beditor scoring system that can be used to select the best performing gRNAs from designed gRNA library. Due to the lack of large scale, genome-wide base editing data, we relied on few general rules that are applicable to all BEs. These rules pertain to the requirements for optimal mutagenesis. With the penalties assigned to each requirement, the beditor score of the optimally performing guide RNA would tend to be higher while that of poorly performing guide RNA would be lower (see Methods). In the future, we wish to determine the values of handcrafted penalty scores from empirical data. The current version of beditor scoring system assesses four of the general requirements for optimal mutagenesis. Based on the conformity of the gRNA to the requirements four penalties scores are assigned (Figure 1b, see Methods). (1) While general rules of the BE mediated editing is an active field of research, we utilize the basic rule which is common between all base editors: the editable base should lie within the maximum activity window of the BE. Thereby, the gRNAs are penalized if the editable base does not lie within the maximum activity window of BE. Next, utilizing the alignments of gRNAs to the genome, potential off-target sites are identified. (2) A penalty score is assigned based on the gRNA binding at off-target. It is evaluated by capturing a general trend of mismatch tolerance along the length of a gRNA (24) (See Methods). Given the lack of large scale, base editing data, we used empirical data from 'conventional' CRISPR-Cas9based genetic screens, assuming that the basic principles of gRNA recruitment and binding would be conserved between the two variants of CRISPR based mutagenesis technologies. (3) Additionally, from alignments of the gRNAs, a penalty is assigned based on the location of the off-target site (genic or intergenic regions). Off-target editing at intergenic regions is less likely to confound the mutational effects compared to functionally important genic regions. Accordingly, penalties are assigned. (4) Lastly, in order to account for number of off-target sites, the 3 penalties are multiplied together to evaluate a beditor score per gRNA. Effectively, the optimal gRNAs have a beditor score of 1 , while a lower beditor score indicate incompatibility with BE requirements, significant off-targets or off-target effects that confound mutational effects. Finally, in order to filter out the gRNAs containing putative RNA polymerase III transcriptional terminators (26), the length of the poly-T stretch per individual gRNA is indicated in the output of beditor.

\section{Demonstrative analysis 1: customizability with respect to base editing strategies}

We demonstrate that the users can incorporate and use custom base editors and PAM recognition sequences by designing gRNA libraries against sets of 1000 randomly assigned mutations (Supplementary methods) with 6 base editors (among which 4 are not yet discovered and are hence called "hypothetical") and 16 PAM recognition sequences. With all the combinations of $\mathrm{BE}$ specific requirements and all combinations of two mutation formats (nucleotide and amino acid) and two modes of mutagenesis 
1 ('remove' and 'create'), gRNA libraries were designed (Figure 2, and Supplementary data 2 1).

\section{Demonstrative analysis 2: ability to work with different species}

To show beditor's ability to work with genomes of different species, we designed gRNA libraries for representative 10 Ensembl genomes, for sets of 1000 randomly assigned mutations (Supplementary methods). The genomes of all the species were directly fetched from the Ensembl Genome database and gRNA libraries were designed for both two mutation formats (nucleotide and amino acid) and two modes of mutagenesis ('remove' and 'create'). Through the designed gRNA libraries (Supplementary data 2) reasonable editability (Table S6) was achieved for all the species.

\section{Case study: designing a gRNA library against a set clinically relevant SNPs}

12 To demonstrate the utility of our computational workflow, we designed a library of gRNAs

13 against a set of clinically relevant SNPs in the human genome composed of 61,083

14 nucleotide level and 81,819 amino acid level mutations (see Supplementary methods).

15 This analysis was carried out with two different BEs: Target-AID and ABE, and two PAM

16 sequences: NGG $(27,28)$ and NG $(29,30)$ and in 'create' and 'reverse' mode. Note that

17 the purpose of this case study analysis is to provide a demonstration of the functionalities

18 of the beditor workflow. For instance, ABE base editor is not known to function in 19 association with NG PAM. Yet, as shown in demonstrative analysis 1, the users may try 20 any combinations of custom base editors and PAM sequences, The output libraries of gRNA sequences (Supplementary data 3 ) targets $\sim 25 \%$ of the total mutations provided as input (Table S7). The resulting gRNA libraries were composed of gRNAs designed with each of the input BEs and PAM sequence which targeted both the strands (Figure 3

$24 \mathrm{a}$ and $\mathrm{b}$ ). On average, $\sim 1.6$ guides were designed for each mutation. Summary 25 visualizations of gRNA libraries (Figure $3 \mathrm{c}$ ) as well as visualizations of alignments of 26 gRNAs with the target sequence (Figure S5) were generated. Additionally, the percentage 27 of substitutions that can be edited with the designed guides (\% editability) is represented 28 as substitution maps (Figure 4) to indicate the proportion of input mutations that can be 29 edited with the input BEs and PAMs. The gRNAs designed for this case study analysis 30 as well as with all the combinations experimentally validated pairs of base editors and 31 PAM sequences (Table S8) are provided as a database with a web interface at 32 http://rraadd88.github.io/soft/beditor.

\section{Performance evaluation of beditor score}

34 From the case study analysis, beditor scores were evaluated for each gRNA sequence in 35 the library. From the distribution of scores (Figure S6), the users may assign a threshold 36 to filter out gRNAs with lower beditor scores. Collectively, by definition, the beditor scores 37 are negatively correlated $(\rho=-0.94)$ with the number of off-target alignments (Figure $5 a$ ) 
1 and penalty assigned for each alignment based on distance of mismatches from the PAM

2 sequence is positively correlated $(\rho=0.65)$ with the distance (Figure $5 b)$. Note that the 3 rank correlation is not perfect because of cases in which there were two mutations in the

4 aligned sequence. Also, purely informed from features of alignments of gRNAs and the 5 requirements of $B E s$, the beditor score recapitulates empirical activity values of gRNAs 6 with a strong positive correlation $(\rho=0.95)$ determined in terms of Cutting Frequency 7 Determination (CFD) score (24) (Figure 5c). 


\section{DISCUSSION}

CRISPR-mediated base editors have recently become a new paradigm in genome editing, owing to their unique ability to carry out precise mutations without the need for DNA breaks (5). Consequently, plethora of applications of BEs have emerged from all corners of the genome editing community, ranging from study of model and non-model organisms $(3,10-13)$ to therapeutics $(14,15)$. However, there has been a lack of robust and customizable software that can design gRNA libraries with any specific requirements of CRISPR-mediated base editing experiments. As presented here, the novel computational workflow of beditor (Figure 1a) fills in this important gap by allowing comprehensive customizability in terms of requirements of BE, PAM sequence and genome and thus increasing the applicability of the CRISPR-mediated base editing technology to broader community of researchers.

We demonstrate the modularity of beditor workflow in terms of the type of BE, PAM and genome by extensively testing them on synthetic sets of mutations. We also show that the workflow can be used to either create a mutation ('create' mode) or remove it ('remove' mode) with support for both nucleotide and amino acid format of mutations. Collectively, therefore, in terms of integrated customizability alone, beditor workflow provides a significant advance over other methods that provided only limited utilities (Table S1). In addition, we also introduce a novel method for a priori estimation of mutagenesis potential of gRNAs (Figure 1b), that utilizes features obtained from off-target alignments such as distance between mismatch and PAM recognition sequence. Such estimations would allow users to select a subset of designed gRNA library that would provide optimal mutagenesis in their experiments.

24 From the case study analysis of $\sim 60,000$ human clinically relevant SNPs, we show that beditor workflow provides all round gRNA design capabilities, scanning though combinations of multiple strategies (Figure $3 a$ and $b$ ). The validations of beditor score from this analysis revealed that rather simple penalty-based evaluations efficiently captured dependence on position of the mismatch in the alignment from the PAM sequence (Figure $5 \mathrm{~b}$ ) and dependence of the off-target effects on number of alignments per gRNA (Figure 5a). Also, the estimations were in strong correlation with empirical data on the off-target effects obtained from 'conventional' CRISPR screens (Figure 5c). This is supported by the currently available data (31), that suggest that the Cas9 induced off target effects could be the predictors of off target effects of BEs (5). However, recent studies have found that the off-target effects of base editors can vary between $A B E$ and CBE (32-34) and could even be largely unpredictable as in the case of CBEs containing APOBEC1 $(33,34)$. Therefore, in future, we wish to update the beditor scoring system further as more information would emerge from large-scale base editing experiments. The

38 future developments of beditor workflow would be carried out in open source manner at 39 https://github.com/rraadd88/beditor. 
1 Together, considering the wide interest in scar-free and precise mutagenesis endowed

2 by CRISPR-mediated base editing, moving ahead, novel and comprehensive gRNA

3 designing workflow of beditor is expected to provide applicability to broad community of

4 researchers and possibly become an essential component of the CRISPR-mediated base

5 editing technology itself.

6 


\section{DATA AVAILABILITY STATEMENT}

2 The authors affirm that all data necessary for confirming the conclusions of this article are

3 represented fully within the article and its tables and figures.

4 Processed data from this study i.e. gRNA libraries designed for demonstrative analysis 51,2 and the case study analysis are provided as Supplementary data 1,2 and 3 6 respectively. This dataset has been deposited using GSA Figshare portal.

7 The beditor software is available at https://github.com/rraadd88/beditor under the GNU 8 General Public Licence (GPLv3). Database of gRNAs designed through beditor workflow 9 can be accessed at http://rraadd88.github.io/soft/beditor.

10 The dataset analyzed in the study i.e. set of clinically associated human mutations were

11 obtained from the Ensembl database in GVF format (ftp://ftp.ensembl.org/pub/release-

12 93/variation/gvf/homo_sapiens/homo_sapiens_clinically_associated.gvf.gz, Date

13 modified: 08/06/2018, 16:13:00).

\section{ACKNOWLEDGEMENT}

16 We thank members of the Landry and Yachie Labs who provided important suggestions 17 and feedback on the manuscript. We would like to thank Hideto Mori from the Yachie Lab 18 for informative discussions.

\section{FUNDING}

21 This work was supported by the Canadian Institutes of Health Research [299432, 324265 22 and 387697 to C.R.L. 364920, 384483 and Frederick Banting and Charles Best graduate 23 scholarship to P.C.D.], and the Japan Society for the Promotion of Science [ S15734 and 24 S17161 to C.R.L. and N.Y.].

\section{CONFLICT OF INTEREST}

27 The authors declare that they have no competing interests. 


\section{REFERENCES}

1. Nishida,K., Arazoe,T., Yachie,N., Banno,S., Kakimoto,M., Tabata,M., Mochizuki,M., Miyabe,A., Araki,M., Hara,K.Y., et al. (2016) Targeted nucleotide editing using hybrid prokaryotic and vertebrate adaptive immune systems. 8729, 1-14.

2. Gaudelli,N.M., Komor,A.C., Rees,H.A., Packer,M.S., Badran,A.H., Bryson,D.I. and Liu,D.R. (2017) Programmable base editing of $A \cdot T$ to $G \cdot C$ in genomic DNA without DNA cleavage. Nature, 551, 464-471.

3. Kim,J.-S. (2018) Precision genome engineering through adenine and cytosine base editing. Nat. plants, 4, 148-151.

4. Hess,G.T., Tycko,J., Yao,D. and Bassik,M.C. (2017) Methods and Applications of CRISPR-Mediated Base Editing in Eukaryotic Genomes. Mol. Cell, 68, 26-43.

5. Rees,H.A. and Liu,D.R. (2018) Base editing: precision chemistry on the genome and transcriptome of living cells. Nat. Rev. Genet., 19, 770-788.

6. Komor,A.C., Kim,Y.B., Packer,M.S., Zuris,J.A. and Liu,D.R. (2016) Programmable editing of a target base in genomic DNA without double-stranded DNA cleavage. Nature, 533, 420.

7. Hess,G.T., Frésard,L., Han,K., Lee,C.H., Li,A., Cimprich,K.A., Montgomery,S.B. and Bassik,M.C. (2016) Directed evolution using dCas9-targeted somatic hypermutation in mammalian cells. Nat. Methods, 13, 1036-1042.

8. Ma,Y., Zhang,J., Yin,W., Zhang,Z., Song,Y. and Chang,X. (2016) Targeted AIDmediated mutagenesis (TAM) enables efficient genomic diversification in mammalian cells. Nat. Methods, 13, 1029-1035.

9. Cox,D.B.T., Gootenberg,J.S., Abudayyeh,O.O., Franklin,B., Kellner,M.J., Joung,J. and Zhang,F. (2017) RNA editing with CRISPR-Cas13 David. Science (80-. )., 0180, 115.

10. Zafra,M.P., Schatoff,E.M., Katti,A., Foronda,M., Breinig,M., Schweitzer,A.Y., Simon,A., Han,T., Goswami,S., Montgomery,E., et al. (2018) Optimized base editors enable efficient editing in cells, organoids and mice. Nat. Biotechnol., 36.

11. Shimatani,Z., Kashojiya,S., Takayama,M., Terada,R., Arazoe,T., Ishii,H., Teramura,H., Yamamoto,T., Komatsu,H., Miura,K., et al. (2017) Targeted base editing in rice and tomato using a CRISPR-Cas9 cytidine deaminase fusion. Nat. Biotechnol., 10.1038/nbt.3833.

12. Tang,W. and Liu,D.R. (2018) Rewritable multi-event analog recording in bacterial and mammalian cells. Science (80-. )., 10, eaap8992.

13. Qin,W., Lu,X. and Lin,S. (2018) Programmable base editing in zebrafish using a modified CRISPR-Cas9 system. Methods, 10.1016/j.ymeth.2018.07.010. 
14. Rossidis,A.C., Stratigis,J.D., Chadwick,A.C., Hartman,H.A., Ahn,N.J., Li,H., Singh,K., Coons,B.E., Li,L., Lv,W., et al. (2018) In utero CRISPR-mediated therapeutic editing of metabolic genes. Nat. Med., 24, 1513-1518.

15. Villiger,L., Grisch-Chan,H.M., Lindsay,H., Ringnalda,F., Pogliano,C.B., Allegri,G., Fingerhut,R., Häberle,J., Matos,J., Robinson,M.D., et al. (2018) Treatment of a metabolic liver disease by in vivo genome base editing in adult mice. Nat. Med., 10.1038/s41591-018-0209-1.

16. Billon,P., Bryant,E.E., Joseph,S.A., Nambiar,T.S., Hayward,S.B., Rothstein,R. and Ciccia,A. (2017) CRISPR-Mediated Base Editing Enables Efficient Disruption of Eukaryotic Genes through Induction of STOP Codons. Mol. Cell, 67, 1068-1079.e4.

17. Nishimasu,H., Yamano,T., Gao,L., Zhang,F., Ishitani,R. and Nureki,O. (2017) Structural Basis for the Altered PAM Recognition by Engineered CRISPR-Cpf1. Mol. Cell, 10.1016/j.molcel.2017.04.019.

18. Zerbino,D.R., Achuthan,P., Akanni,W., Amode,M.R., Barrell,D., Bhai,J., Billis,K., Cummins,C., Gall,A., Girón,C.G., et al. (2018) Ensembl 2018. Nucleic Acids Res., 10.1093/nar/gkx1098.

19. PyEnsembl (2018) https://github.com/openvax/pyensembl.

20. Quinlan,A.R. and Hall,I.M. (2010) BEDTools: A flexible suite of utilities for comparing genomic features. Bioinformatics, 10.1093/bioinformatics/btq033.

21. Li,H. and Durbin,R. (2009) Fast and accurate short read alignment with BurrowsWheeler transform. Bioinformatics, 10.1093/bioinformatics/btp324.

22. Li,H., Handsaker,B., Wysoker,A., Fennell,T., Ruan,J., Homer,N., Marth,G., Abecasis,G. and Durbin,R. (2009) The Sequence Alignment/Map format and SAMtools. Bioinformatics, 25, 2078-2079.

23. Concordet,J.-P. and Haeussler,M. (2018) CRISPOR: intuitive guide selection for CRISPR/Cas9 genome editing experiments and screens. Nucleic Acids Res., 46, W242-W245.

24. Doench,J.G., Fusi,N., Sullender,M., Hegde,M., Vaimberg,E.W., Donovan,K.F., Smith,I., Tothova,Z., Wilen,C., Orchard,R., et al. (2016) Optimized sgRNA design to maximize activity and minimize off-target effects of CRISPR-Cas9. Nat. Biotechnol., 34, 184-191.

25.

Ensembl:

Table

of

Assemblies. https://useast.ensembl.org/info/website/archives/assembly.html (2018) Accessed on 18 Aug 2018.

26. Gao,Z., Herrera-Carrillo,E. and Berkhout,B. (2018) Delineation of the Exact Transcription Termination Signal for Type 3 Polymerase III. Mol. Ther. - Nucleic Acids, 10.1016/j.omtn.2017.11.006. 
27. Hsu,P.D., Scott,D.A., Weinstein,J.A., Ran,F.A., Konermann,S., Agarwala,V., Li,Y., Fine,E.J., Wu,X., Shalem,O., et al. (2013) DNA targeting specificity of RNA-guided Cas9 nucleases. Nat. Biotechnol., 31, 827-832.

28. Jinek,M., Chylinski,K., Fonfara,I., Hauer,M., Doudna,J.A. and Charpentier,E. (2012) A programmable dual-RNA-guided DNA endonuclease in adaptive bacterial immunity. Science (80-. )., 10.1126/science.1225829.

29. Hu,J.H., Miller,S.M., Geurts,M.H., Tang,W., Chen,L., Sun,N., Zeina,C.M., Gao,X., Rees,H.A., Lin,Z., et al. (2018) Evolved Cas9 variants with broad PAM compatibility and high DNA specificity. Nature, 556, 57-63.

30. Nishimasu,H., Shi,X., Ishiguro,S., Gao,L., Hirano,S., Okazaki,S., Noda,T., Abudayyeh,O.O., Gootenberg,J.S., Mori,H., et al. (2018) Engineered CRISPR-Cas9 nuclease with expanded targeting space. Science (80-. )., 9129, eaas9129.

31. Kim,D., Lim,K., Kim,S.T., Yoon,S.H., Kim,K., Ryu,S.M. and Kim,J.S. (2017) Genomewide target specificities of CRISPR RNA-guided programmable deaminases. Nat. Biotechnol., 35, 475-480.

32. Lee,H.K., Willi,M., Miller,S.M., Kim,S., Liu,C., Liu,D.R. and Hennighausen,L. (2018) Targeting fidelity of adenine and cytosine base editors in mouse embryos. Nat. Commun., 10.1038/s41467-018-07322-7.

33. Jin,S., Zong,Y., Gao,Q., Zhu,Z., Wang,Y., Qin,P., Liang,C., Wang,D., Qiu,J.-L., Zhang,F., et al. (2019) Cytosine, but not adenine, base editors induce genome-wide off-target mutations in rice. Science (80-. )., 10.1126/science.aaw7166.

34. Zuo,E., Sun,Y., Wei,W., Yuan,T., Ying,W., Sun,H., Yuan,L., Steinmetz,L.M., Li,Y. and Yang,H. (2019) Cytosine base editor generates substantial off-target singlenucleotide variants in mouse embryos. Science (80-. )., 126, eaav9973. 


\section{TABLE AND FIGURES LEGENDS}

Figure 1. The computational workflow of beditor allows for the flexible design of gRNA libraries to be used in CRISPR base editing and offers a priori evaluation of mutagenesis potential.

a Information on the type and location of desired mutations is supplied to the beditor workflow as a tab separated file. gRNAs are designed according to the user provided sets of BEs and PAM recognition sequences. Among many base editor and Pam sequence specific requirements, nucleotide windows for maximum activity are considered while designing the gRNAs. Finally, potential off-target effects are estimated.

b A scoring system specifically designed for a priori evaluation of mutagenesis potential of gRNAs. Penalties are assigned based on (1) the total number of off-target alignments of gRNAs to the reference genome, (2) positions of the mismatches in the off-target alignments relative to the PAM and (3) genomic locations of off-target alignments and lastly, (4) whether the editable base lies inside the activity window of the BE. Using all of the above penalties, a final score is calculated for each gRNA sequence - the beditor score.

Figure 2. Demonstrative analysis of gRNAs designed with custom base editors and PAM recognition sequences.

21

22

In order to demonstrate the utility of beditor in utilizing custom base editors and PAM recognition sequences, sets of 1000 randomly assigned mutations in S. cerevisiae (see Supplementary methods) were analyzed in 2 mutation formats (nucleotide or amino acid) and 2 modes of mutagenesis ("create" and "remove") (each combination is shown in panel $\mathbf{a}$ to $\mathbf{d}$ ). In each heatmap, number of gRNAs designed by each combination of a base editor (in columns) and PAM recognition sequence (in rows) is shown.

Figure 3. Case study analysis of clinically relevant human SNPs.

29

For the case study analysis, 2 base-editors (Target-AID and ABE) and 2 PAM sequences (NGG and NG) were used. Number of gRNAs designed using each mutation format i.e. nucleotide (a) and amino acid mutation (b) data are shown.

c Representative summary visualization of gRNA libraries designed with Target-AID base editor. Nucleotide composition of the gRNAs is shown along the length of the gRNAs. gRNAs are grouped by the position of the editable nucleotides within the activity window of a $B E$ (shown in the rows).

Figure 4. Percentage editability of gRNA libraries designed in case study analysis of clinically relevant human SNPs.

Percentage of substitutions that can be edited by gRNA library (\% editability) designed for case study analysis of clinically relevant human SNPs, in the format of nucleotide (a and b) and amino acid mutations (c and $\mathbf{d}$ ) (see Supplementary methods). Also, the gRNA libraries were designed to remove mutations i.e. 'remove' mode (a and $\mathbf{c}$ ) and to 43 introduce mutations i.e. 'create' mode ( $\mathbf{b}$ and $\mathbf{d}$ ). Mapped on the heatmaps is a ratio 
1 between number of substitutions that can be edited with the designed gRNAs and the 2 number of substitutions present in the input data (\% editability). Left and right brackets 3 indicate that the substitution is carried out by ABE and Target-AID respectively. +, - and $4 \pm$ indicate substitutions for which gRNA is designed on + , - and both strands 5 respectively. Shown in gray are substitutions that are absent in the input data. * 6 indicates non-sense mutation.

Figure 5. Performance assessment of beditor scores from case study analysis of 10 clinically relevant human SNPs.

11 a Relationship between the number of genome-wide off-target alignments and beditor score per gRNA. The color of hexbins are scaled according to the number of gRNAs per bin.

b Relationship between the distance of a mutation in off-target alignments and corresponding penalty assigned $\left(P_{a}\right)$. The color of hexbins are scaled according to the number of off-target alignments per bin.

c Relationship between the CFD score and beditor score for all the gRNAs carrying NGG PAM sequence. The color of hexbins are scaled according to the number of gRNAs per

$20 \rho$ is Spearman's correlation coefficient. 


\section{Figure 1}

\section{General workflow of beditor}

Input (1) Type of mutation

nucleotide/amino acid

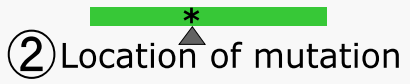

Guide

design

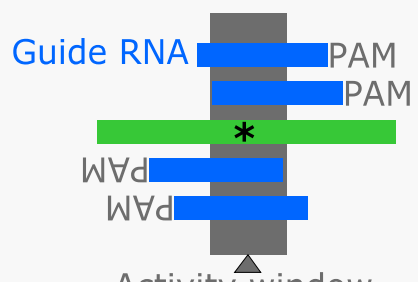

Activity window

of base editor

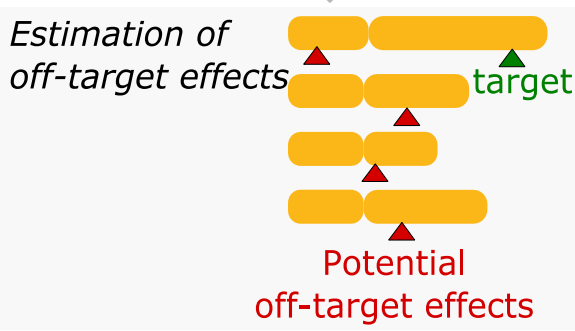

$\mathrm{b}^{\boldsymbol{A} \text { priori evaluation of mutagenesis potential }}$
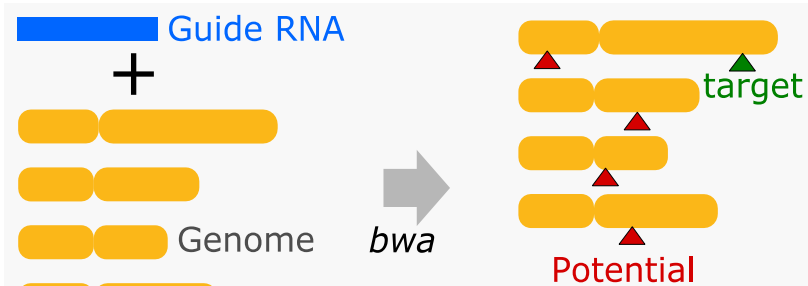

off-target effects

(1)

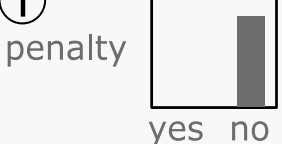

within activity window of the base editor

Location of base to be edited

(3)

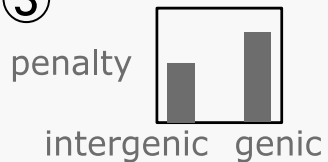

Locations of

off-target alignments
(2)

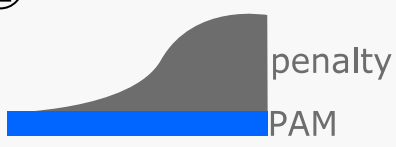

Locations of mismatches in off-target alignments relative to PAM sequence

(4)

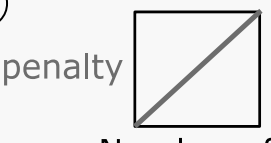

Number of

off-target alignments 


\section{$1 \quad$ Figure 2}

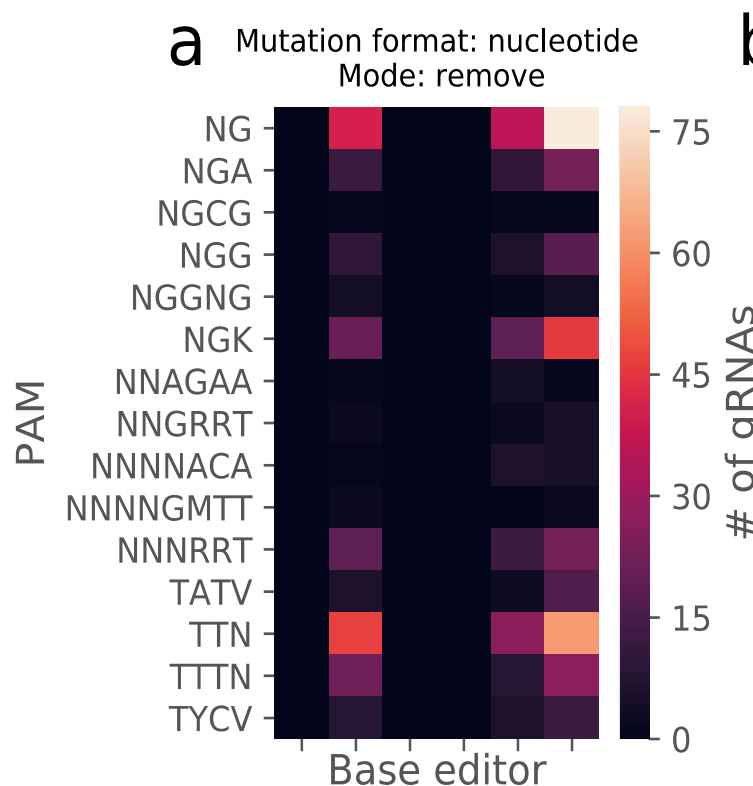

C Mutation format: amino acid Mode: remove

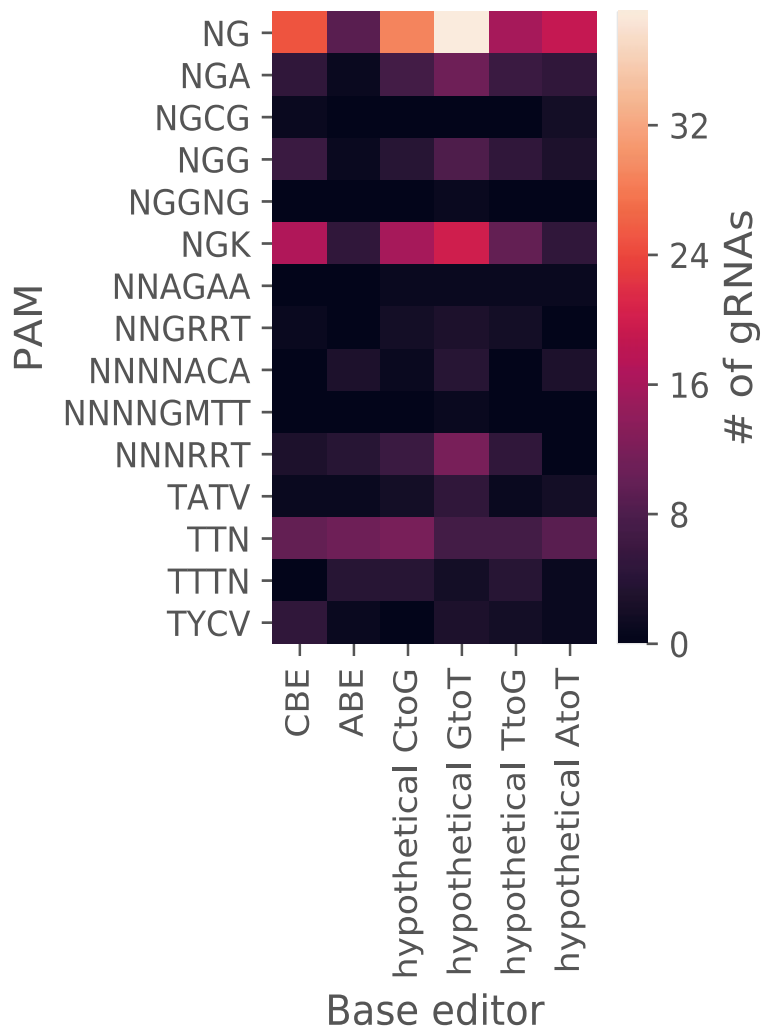

Base editor b Mutation format: nucleotide Mode: create

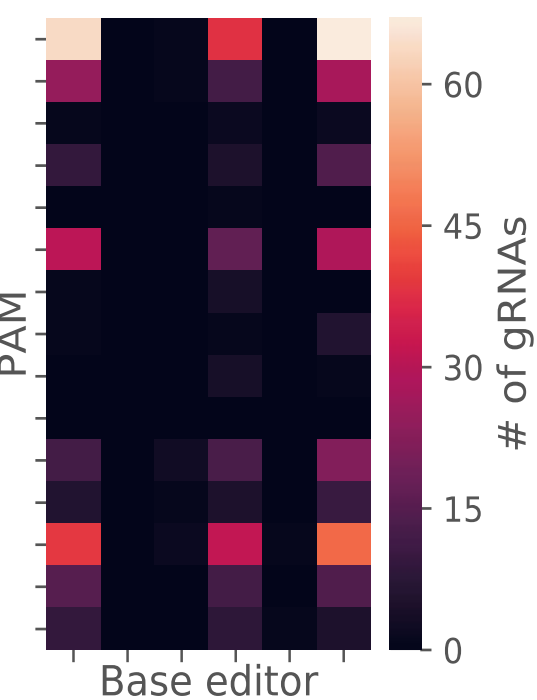

Mutation format: amino acid Mode: create

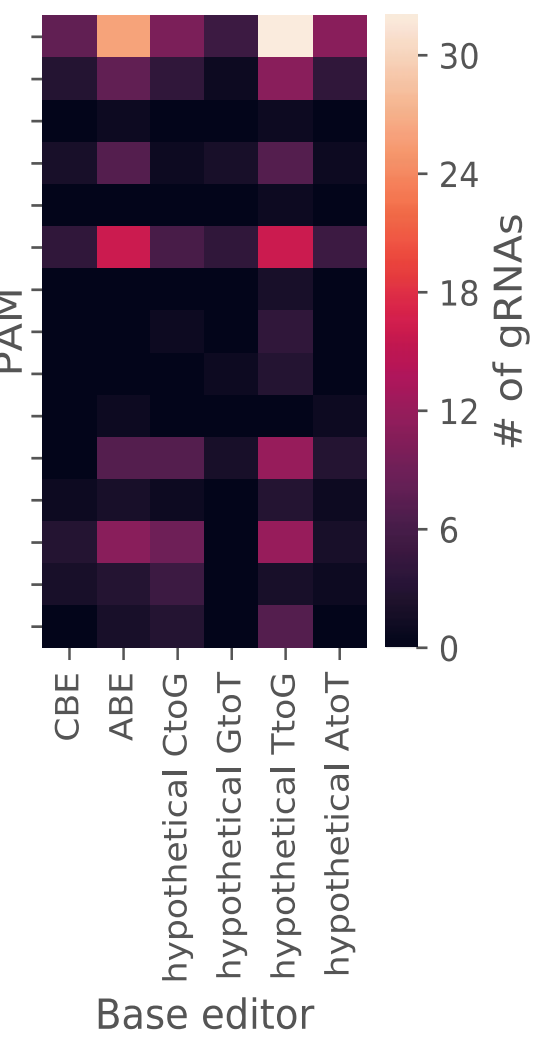


bioRxiv preprint doi: $h t t p s: / / d o i . o r g / 10.1101 / 426973$; this version posted March 8 , 2019. The copyright holder for this preprint (which was not certified by peer review) is the author/funder, who has granted bioRxiv a license to display the preprint in perpetuity. It is made available under aCC-BY-NC-ND 4.0 International license.

1

\section{Figure 3}

a Nucleotide mutations

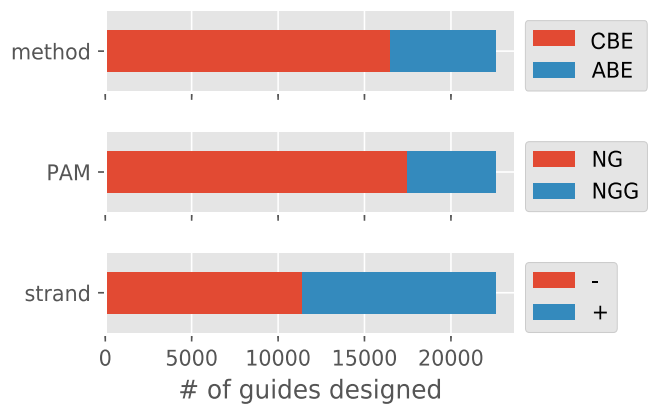

b Amino acid mutations

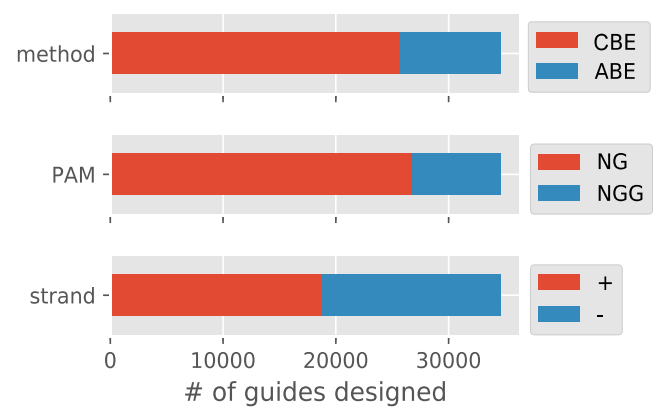

\section{Visualization of gRNA libraries}

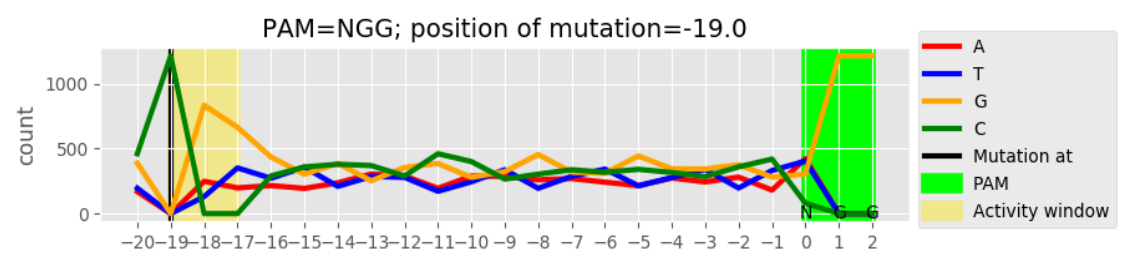

$\mathrm{PAM}=\mathrm{NGG}$; position of mutation $=-18.0$

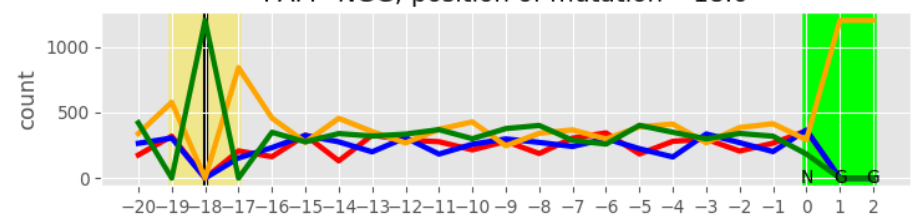

PAM=NGG; position of mutation $=-17.0$

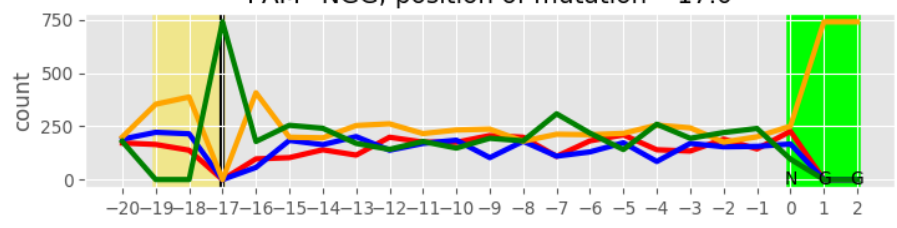


bioRxiv preprint doi: https://doi org/10.1101/426973; this version posted March 8,2019 . The copyright holder for this preprint (which was not certified by peer review) is the author/funder, who has granted bioRxiv a license to display the preprint in perpetuity. It is made available under aCC-BY-NC-ND 4.0 International license.

\section{$1 \quad$ Figure 4}

a Mutation format : nucleotide Mode: remove

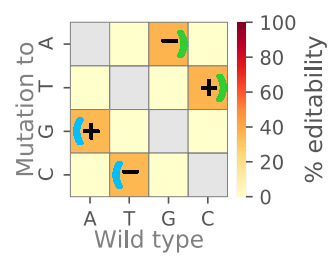

C Mutation format : amino acid Mode: remove

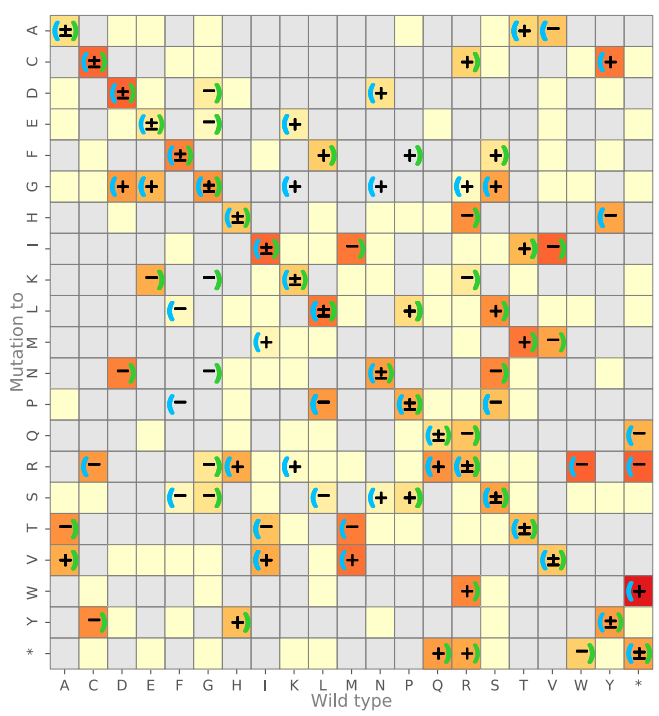

b Mutation format : nucleotide Mode: create

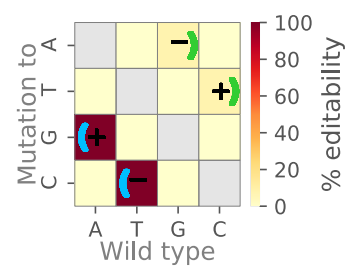

d Mutation format : amino acid Mode: create
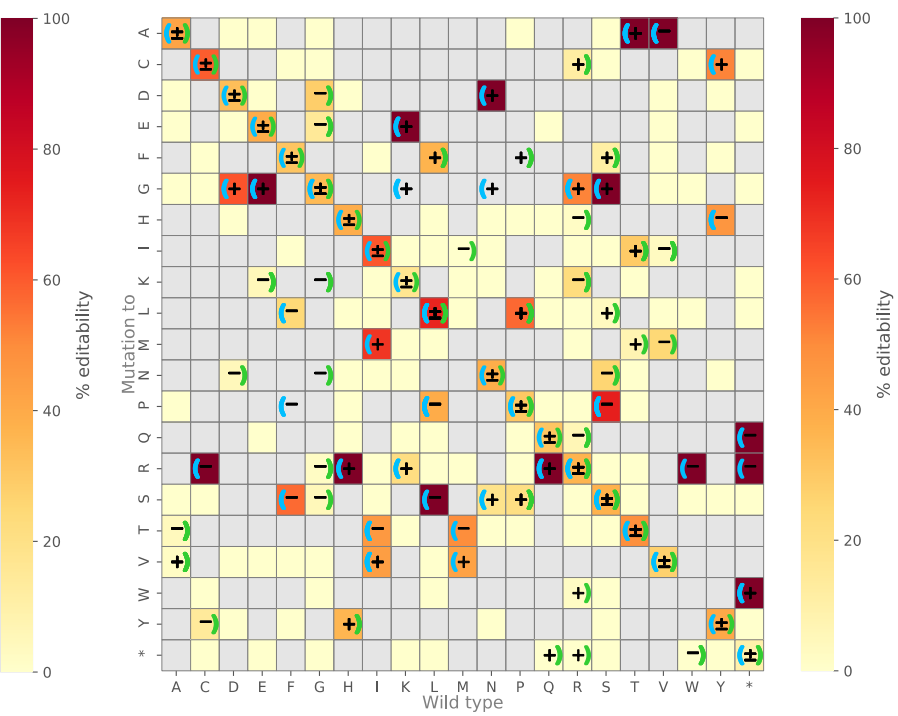


\section{Figure 5}
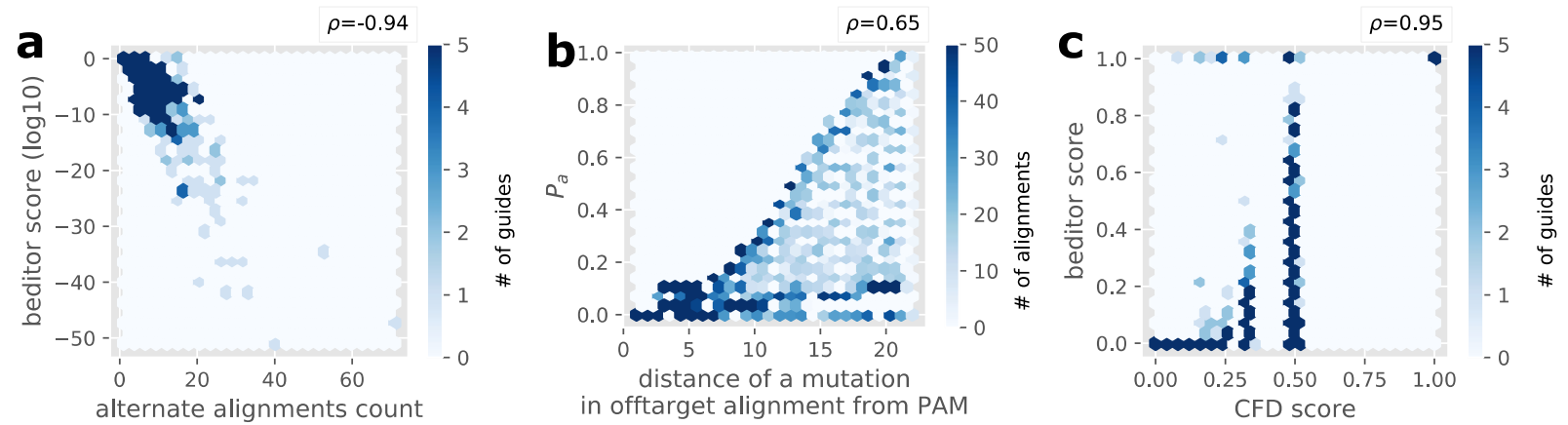

2 


\section{Supporting information}

\section{beditor. A computational workflow for designing libraries of guide RNAs for CRISPR-mediated base editing}

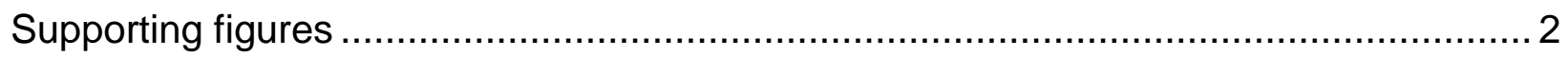

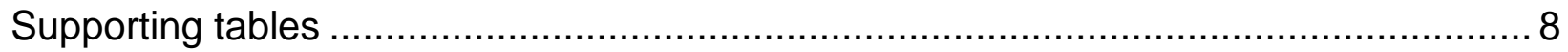

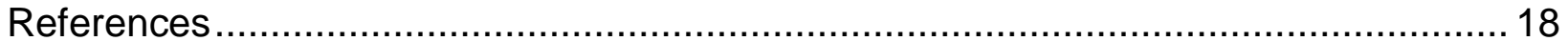




\section{Supporting figures}

a Nucleotides

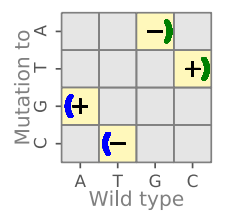

b

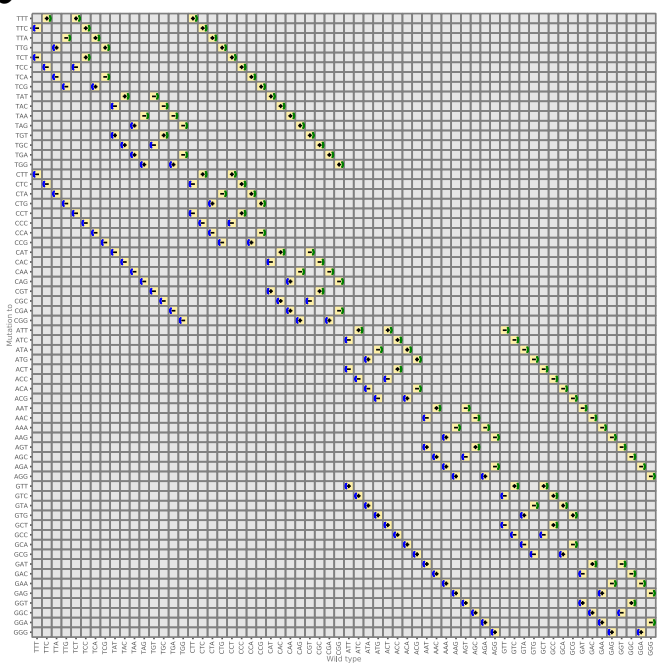

C
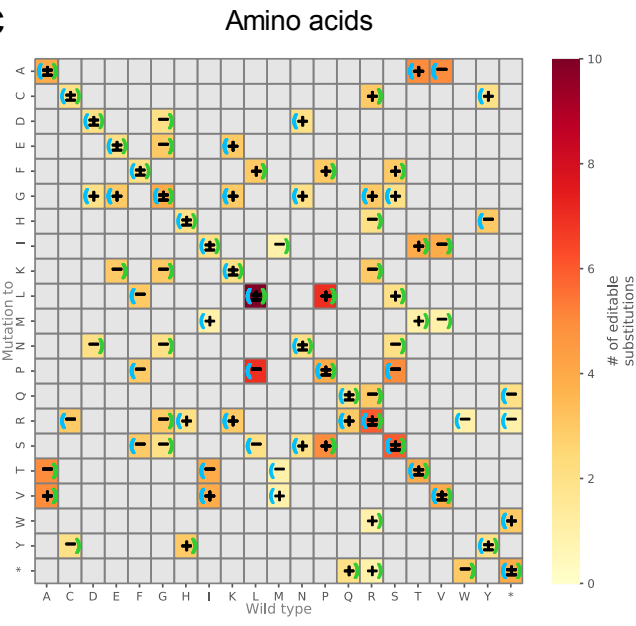

Flg $S 1$ : Possible substitutions by current ABE and CBE.

a Nucleotide level substitutions.

b Codon level substitutions.

b Amino acid level substitutions.

Shown on the heatmaps are the cumulative number of substitutions that can be edited with either $\mathrm{ABE}$ or $\mathrm{CBE}$. Left and right brackets indicate that the substitution is carried out by $A B E$ and $C B E$ respectively. + , - and \pm indicate substitutions for which guide RNA is designed on the,+- and both the strands respectively. Shown in gray are substitutions that are absent in the input data. The symbol * represents a non-sense mutation. 


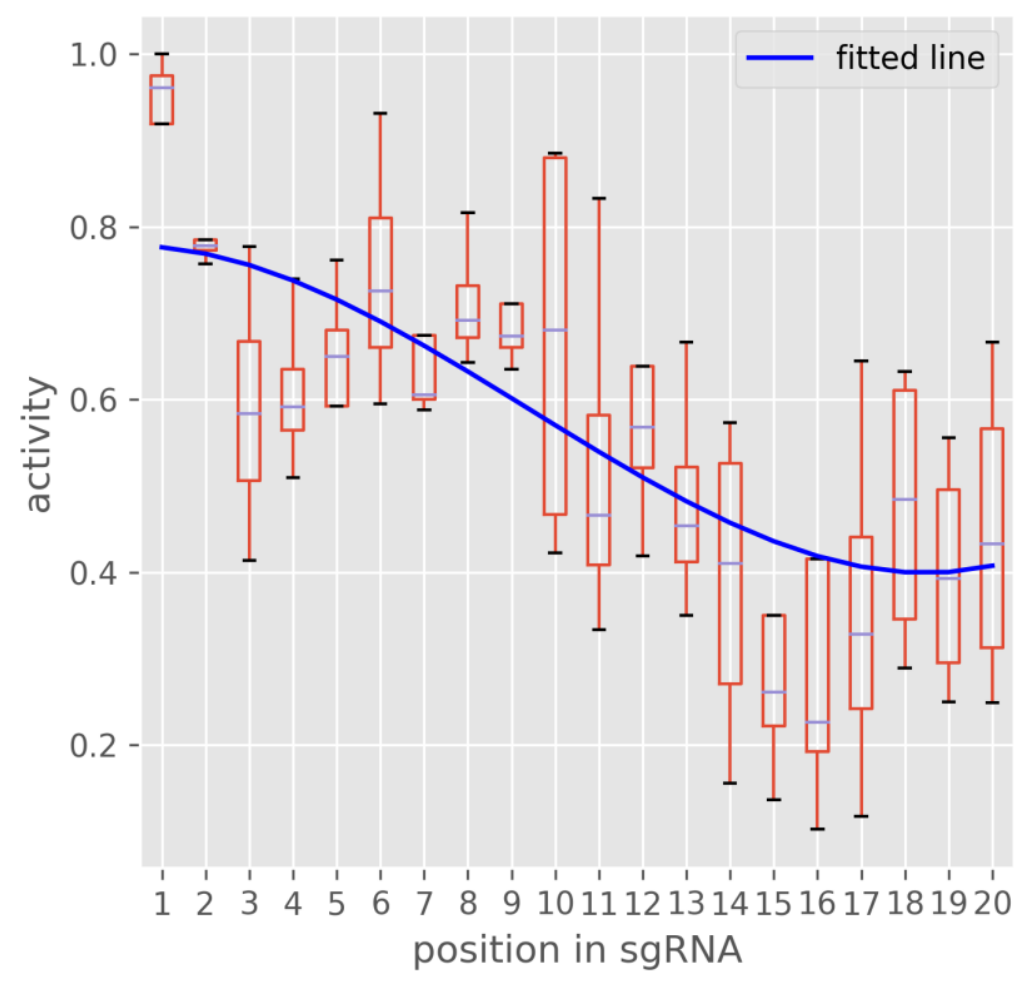

Fig S2: Fitting a third degree polynomial equation to the mismatch tolerance data from Doench et. al (3).

The gRNA activity values at each mismatch position are shown as boxplots (red). The third degree polynomial equation fitted line is shown in blue. 

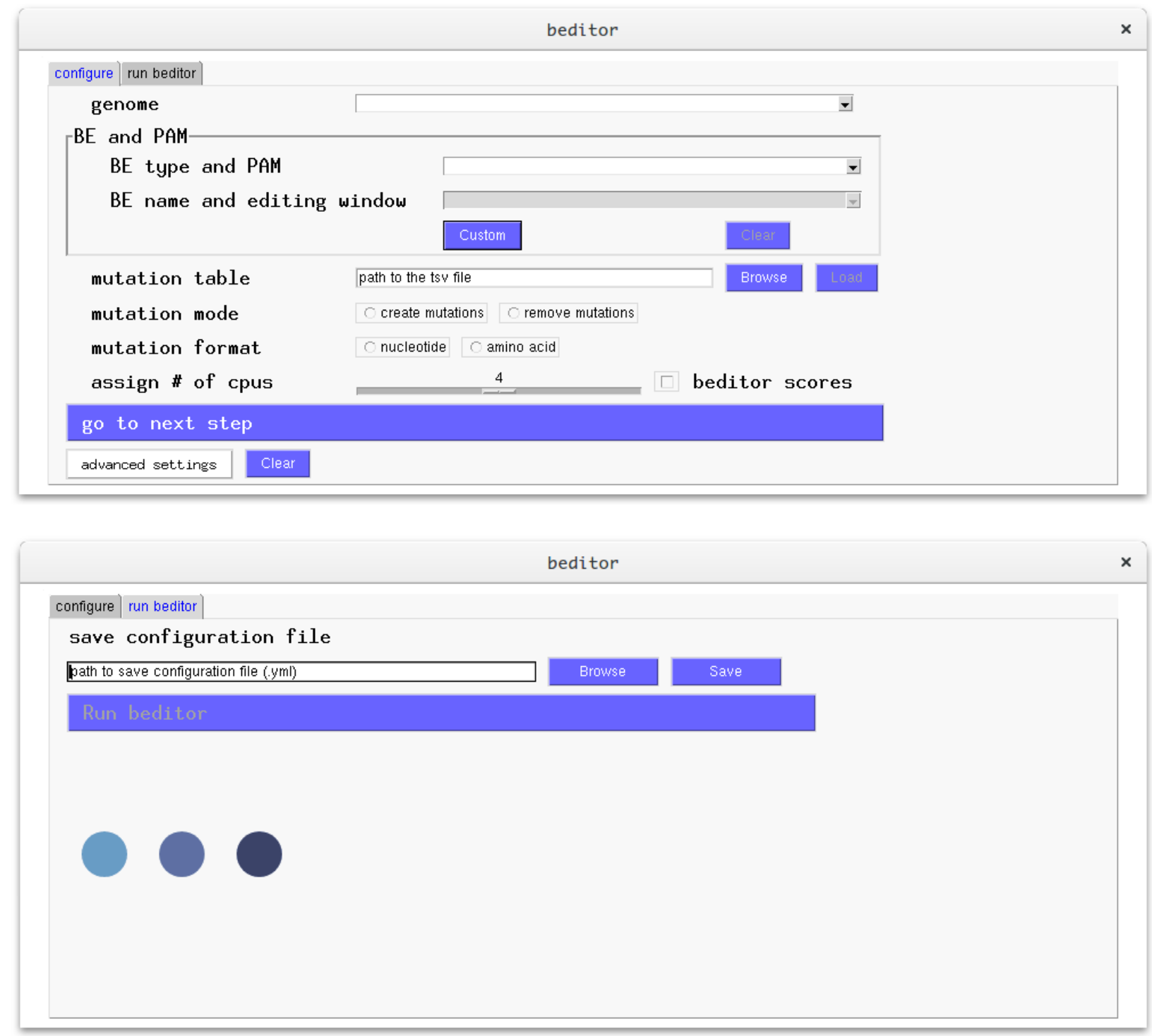

Fig S3: Graphical User Interface (GUI) of beditor.

Top: In the first tab of the GUI, the parameters for the analysis workflow can be provided using a series of options. These basic parameters include the species name, the name of the base editor and the PAM sequence, an input list of mutations, the format of mutations (amino acids and nucleotide) and mode of mutagenesis (create or remove), the number of cores (processors) and If the beditor scores are to be calculated for the gRNAs.

Bottom: the second tab of the GUI provides option to save the parameters of the analysis workflow as a YAML file and then subsequently run the program. 

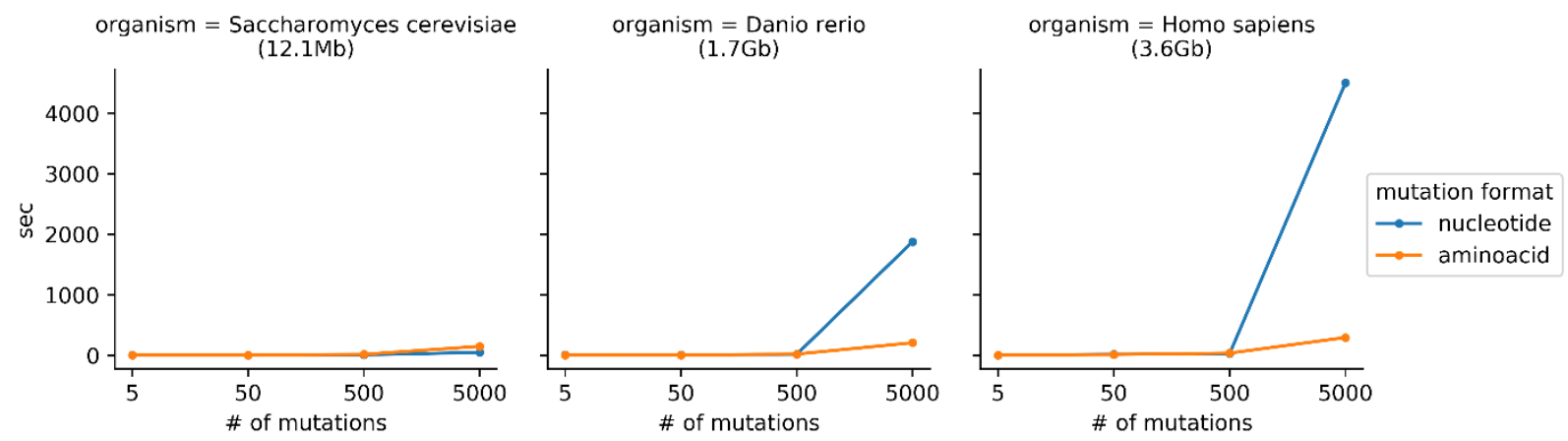

Fig S4: The time taken to design gRNA libraries depends on the number of mutations and the size of the genome.

Sets of 5-5000 nucleotide and amino acid mutations were tested with genomes of 3 species. 6 parallel processors (cores) were used for the analysis. The analysis was carried out using test_beditor.py script from test_beditor repository (https://github.com/rraadd88/test beditor). Note that the time does not include the time taken for installation of the genomes (i.e. downloading and indexing of the genomes). 


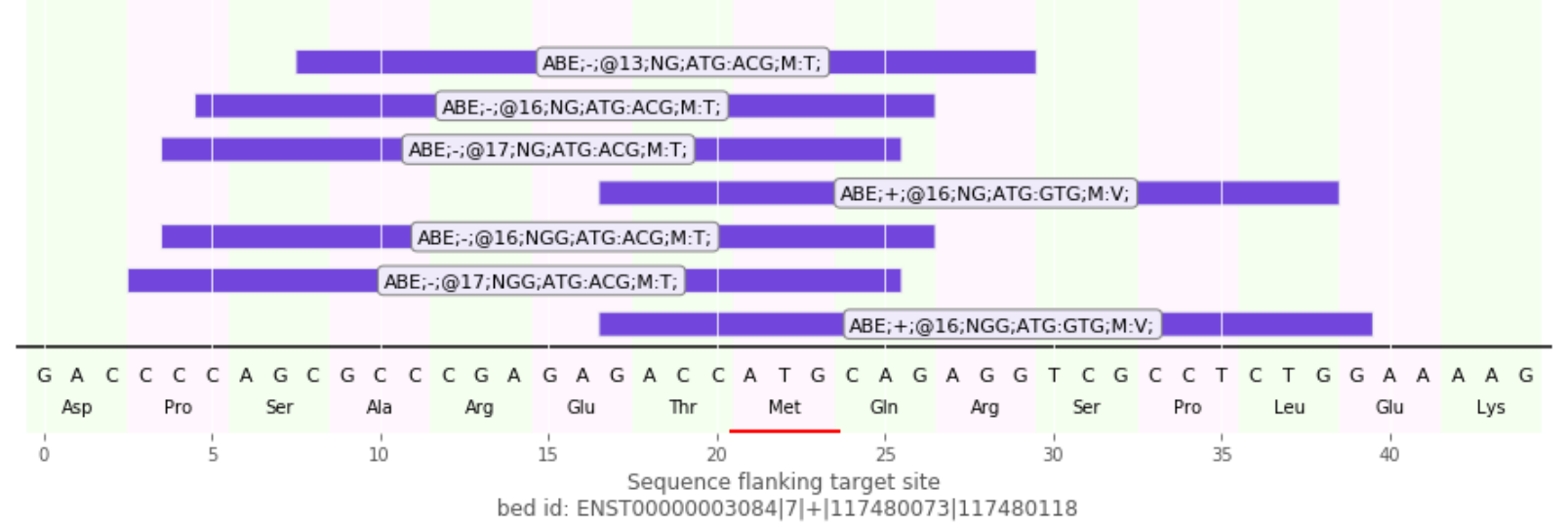

Fig S5: Representative visualizations of alignment between a gRNA and target genomic sequence.

The gRNA are shown in purple and with annotations denoting its identity (name of the base editor, target strand, distance from PAM sequence, PAM, reference codon, mutated codon, wild-type amino acid and mutated amino acid) is shown on the guide RNA. The target site is indicated in red color. Reading frames and genomic coordinates of the target DNA are shown below. 


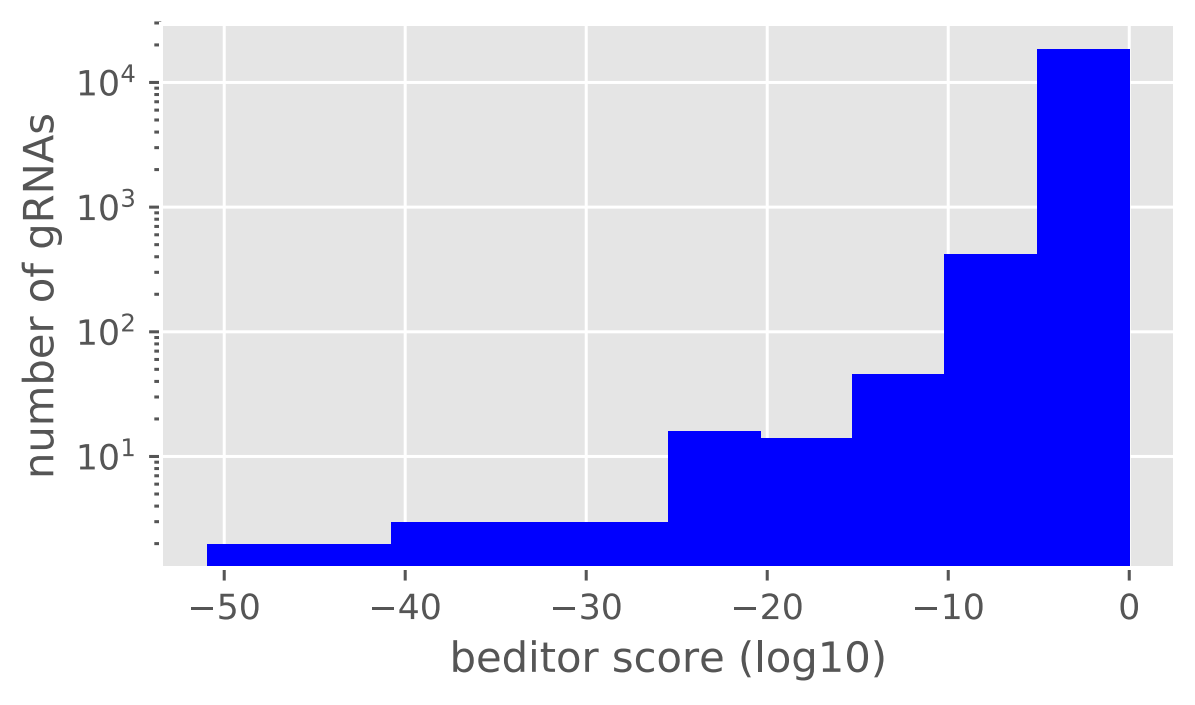

Fig S6: Distribution of beditor scores for the gRNA library designed for the demonstrative analysis of clinically associated human nucleotide mutations in 'create' mode. 


\section{Supporting tables}

Table S1: Comparison of features of beditor with other existing tools.

\begin{tabular}{|c|c|c|c|c|}
\hline Feature & beditor & iSTOP [1] & $\begin{array}{l}\text { BE- } \\
\text { analyzer[2] }\end{array}$ & Benchling [3] \\
\hline $\begin{array}{l}\text { Number of species whose genomes } \\
\text { are directly compatible }\end{array}$ & $>125$ & $\sim 70$ & $\sim 40$ & $>100$ \\
\hline $\begin{array}{l}\text { Searches sites editable by base } \\
\text { editors }\end{array}$ & Yes & Yes & Yes & $\begin{array}{l}\text { Yes only BE } \\
{[4]}\end{array}$ \\
\hline $\begin{array}{l}\text { Designs gRNAs against predefined } \\
\text { set of nucleotide mutations }\end{array}$ & Yes & No & No & No \\
\hline $\begin{array}{l}\text { Designs gRNAs against predefined } \\
\text { set of amino acid mutations }\end{array}$ & Yes & No & No & No \\
\hline $\begin{array}{l}\text { Designs gRNAs for mutational } \\
\text { scanning using a predefined amino } \\
\text { acid substitution matrix }\end{array}$ & Yes & No & No & No \\
\hline $\begin{array}{l}\text { Designs gRNAs against non-sense } \\
\text { mutations }\end{array}$ & Yes & Yes & No & Yes \\
\hline Designs control gRNAs & Yes & No & No & No \\
\hline $\begin{array}{l}\text { Generation of gRNA libraries for } \\
\text { genome-wide screening of } \\
\text { mutations }\end{array}$ & Yes & No & $\begin{array}{l}1 \text { target site at } \\
\text { a time }\end{array}$ & $\begin{array}{l}1 \text { target site } \\
\text { at a time }\end{array}$ \\
\hline Multithreading/ parallel computing & Yes & No & No & No \\
\hline $\begin{array}{l}\text { Allows addition of any novel/custom } \\
\text { PAM recognition sequence }\end{array}$ & Yes & No & No & No \\
\hline $\begin{array}{l}\text { Allows addition of any novel/custom } \\
\text { base editors }\end{array}$ & Yes & No & No & No \\
\hline $\begin{array}{l}\text { Estimation of editing efficiency of } \\
\text { gRNAs }\end{array}$ & Yes & No & No & Yes \\
\hline $\begin{array}{l}\text { Integrates independent measures of } \\
\text { off-target effects }\end{array}$ & $\begin{array}{l}\text { Yes } \\
\text { (CFD } \\
\text { scores[5]) }\end{array}$ & No & No & Yes \\
\hline
\end{tabular}


bioRxiv preprint doi: https://doi.org/10.1101/426973; this version posted March 8, 2019. The copyright holder for this preprint (which was not certified by peer review) is the author/funder, who has granted bioRxiv a license to display the preprint in perpetuity. It is made available under aCC-BY-NC-ND 4.0 International license.

Table S2: Penalties used for the calculation of the beditor score.

\begin{tabular}{|l|l|}
\hline Penalty & Value \\
\hline $\boldsymbol{P}_{\min }$ & 0.9 \\
\hline $\boldsymbol{P}_{\max }$ & 0.1 \\
\hline $\boldsymbol{G}_{\mathrm{g}}$ & 0.5 \\
\hline $\boldsymbol{G}_{\text {ig }}$ & 0.9 \\
\hline $\boldsymbol{A}$ & 0.5 \\
\hline
\end{tabular}


Table S3: Default PAM recognition sequences supported by beditor.

\begin{tabular}{|l|l|c|c|l|}
\hline PAM & Description & position & $\begin{array}{l}\text { length of } \\
\text { guide } \\
\text { sequence }\end{array}$ & Reference \\
\hline NG & xCas9 & $3^{\prime}$ & 20 & {$[6]$} \\
\hline NGA & SpCas9 mutant & $3^{\prime}$ & 20 & {$[7]$} \\
\hline NGCG & SpCas9 mutant & $3^{\prime}$ & 20 & {$[7]$} \\
\hline NGG & SpCas9 & $3^{\prime}$ & 20 & {$[8,9]$} \\
\hline NGGNG & Cas9 S. Thermophilus & $3^{\prime}$ & 20 & {$[10,11]$} \\
\hline NGK & xCas9 & $3^{\prime}$ & 20 & {$[6]$} \\
\hline NGN & xCas9 & $3^{\prime}$ & 20 & {$[6]$} \\
\hline NNAGAA & Cas9 S. Thermophilus & $3^{\prime}$ & 20 & {$[10,11]$} \\
\hline NNGRRT & SaCas9 & $3^{\prime}$ & 21 & {$[12]$} \\
\hline NNNNACA & CjCas9 & $3^{\prime}$ & 20 & {$[13]$} \\
\hline NNNNGMTT & Cas9 N. Meningitidis & $3^{\prime}$ & 20 & {$[14]$} \\
\hline NNNRRT & KKH SaCas9 & $3^{\prime}$ & 21 & {$[7,12]$} \\
\hline TATV & AsCpf1 mutant & $5^{\prime}$ & 23 & {$[15]$} \\
\hline TTN & Cpf1 F. Novicida & $5^{\prime}$ & 23 & {$[16]$} \\
\hline TTTN & Cpf1 Acidaminococcus / & $5^{\prime}$ & 23 & {$[16]$} \\
\hline TYCV & Lachnospiraceae & $5^{\prime}$ & 23 & {$[15]$} \\
\hline
\end{tabular}




\section{Table S4: Input parameters used for the demonstrative analysis of clinically associated human genetic variants}

\begin{tabular}{|c|c|c|}
\hline Variable & Input & Description \\
\hline Host & & Name of host organism \\
\hline genomerelease & 93 & Ensembl genome release \\
\hline genomeassembly & & Genome assembly version \\
\hline dinp & din.tsv & File path of input tab-separated file \\
\hline mutation_format & $\begin{array}{l}\text { [aminoacid, } \\
\text { nucleotide] }\end{array}$ & $\begin{array}{l}\text { Whether the input data consists of amino acid or } \\
\text { nucleotide mutations. }\end{array}$ \\
\hline reverse_mutations & [FALSE, TRUE] & $\begin{array}{l}\text { FALSE if design guide RNAs to 'create' mutations, } \\
\text { TRUE to 'remove' mutations. }\end{array}$ \\
\hline Mutations & mutations & $\begin{array}{l}\text { Information about mutated amino acid is taken from the } \\
\text { input file }\end{array}$ \\
\hline mutation_type & $\mathrm{N}$ & $\begin{array}{l}\text { Type of mutations to process, N: non-synonymous, S: } \\
\text { synonymous, else: both }\end{array}$ \\
\hline keep_mutation_nonsense & FALSE & Whether to process non-sense mutations \\
\hline pams & [NGG, NG] & List of PAMs to use \\
\hline bes & [Target-AID, ABE] & List of Base Editors to use \\
\hline max_subs_per_codon & 1 & $\begin{array}{l}\text { Maximum number of nucleotides that can be edited in } \\
\text { the target codon. }\end{array}$ \\
\hline mismatches_max & 2 & $\begin{array}{l}\text { Maximum number of mismatches allowed in the } \\
\text { alignment gRNAs against reference genome. }\end{array}$ \\
\hline Cores & 5 & Number of processors to use for parallel processing \\
\hline Chunksize & 200 & Number of mutations to process per processor \\
\hline
\end{tabular}


Table S5: Summary statistics of demonstrative analysis with custom base editors and PAM recognition sequences.

\begin{tabular}{|l|r|r|r|r|}
\hline & nucleotide & $\begin{array}{l}\text { amino } \\
\text { acid }\end{array}$ & nucleotide & \multicolumn{1}{l|}{$\begin{array}{l}\text { amino } \\
\text { acid }\end{array}$} \\
\hline Remove/create mutation & \multicolumn{1}{|l}{ remove } & remove & \multicolumn{1}{l|}{ create } & \multicolumn{1}{l|}{ create } \\
\hline $\begin{array}{l}\text { Total number of mutations in the input } \\
\text { data }\end{array}$ & 1000 & 1000 & 1000 & 1000 \\
\hline Total number of mutations edited & 263 & 117 & 246 & 105 \\
\hline Total number of guides designed & 817 & 562 & 791 & 403 \\
\hline \% editability & 26.3 & 11.7 & 24.6 & 10.5 \\
\hline number of guides per mutation & 3.10646 & 4.80342 & 3.21545 & 3.8381 \\
\hline
\end{tabular}


Table S6: Summary statistics of demonstrative analysis of representative set of species.

\begin{tabular}{|c|c|c|c|c|c|}
\hline Species & metrics & $\begin{array}{l}\text { nucleoti } \\
\text { de }\end{array}$ & $\begin{array}{l}\text { amino } \\
\text { acid }\end{array}$ & $\begin{array}{l}\text { nucleoti } \\
\text { de }\end{array}$ & $\begin{array}{l}\text { amino } \\
\text { acid }\end{array}$ \\
\hline & Remove/create mutation & remove & remove & create & create \\
\hline & $\begin{array}{l}\text { Total number of mutations in the input } \\
\text { data }\end{array}$ & 1000.00 & 1000.00 & 1000.00 & 1000.00 \\
\hline \multirow[t]{4}{*}{ Bos taurus } & Total number of mutations edited & 76.00 & 45.00 & 69.00 & 36.00 \\
\hline & Total number of guides designed & 123.00 & 83.00 & 121.00 & 62.00 \\
\hline & \% editability & 7.60 & 4.50 & 6.90 & 3.60 \\
\hline & number of guides per mutation & 1.62 & 1.84 & 1.75 & 1.72 \\
\hline \multirow[t]{4}{*}{ Danio rerio } & Total number of mutations edited & 45.00 & 39.00 & 57.00 & 43.00 \\
\hline & Total number of guides designed & 71.00 & 59.00 & 74.00 & 72.00 \\
\hline & $\%$ editability & 4.50 & 3.90 & 5.70 & 4.30 \\
\hline & number of guides per mutation & 1.58 & 1.51 & 1.30 & 1.67 \\
\hline \multirow[t]{4}{*}{ Equus caballus } & Total number of mutations edited & 80.00 & 53.00 & 55.00 & 43.00 \\
\hline & Total number of guides designed & 136.00 & 94.00 & 92.00 & 81.00 \\
\hline & \% editability & 8.00 & 5.30 & 5.50 & 4.30 \\
\hline & number of guides per mutation & 1.70 & 1.77 & 1.67 & 1.88 \\
\hline \multirow[t]{4}{*}{ Felis catus } & Total number of mutations edited & 94.00 & 55.00 & 81.00 & 50.00 \\
\hline & Total number of guides designed & 168.00 & 103.00 & 160.00 & 80.00 \\
\hline & \% editability & 9.40 & 5.50 & 8.10 & 5.00 \\
\hline & number of guides per mutation & 1.79 & 1.87 & 1.98 & 1.60 \\
\hline \multirow[t]{4}{*}{ Gallus gallus } & Total number of mutations edited & 93.00 & 39.00 & 74.00 & 46.00 \\
\hline & Total number of guides designed & 180.00 & 71.00 & 139.00 & 77.00 \\
\hline & \% editability & 9.30 & 3.90 & 7.40 & 4.60 \\
\hline & number of guides per mutation & 1.94 & 1.82 & 1.88 & 1.67 \\
\hline \multirow{4}{*}{$\begin{array}{l}\text { Macaca } \\
\text { fascicularis }\end{array}$} & Total number of mutations edited & 45.00 & 51.00 & 52.00 & 48.00 \\
\hline & Total number of guides designed & 89.00 & 83.00 & 90.00 & 75.00 \\
\hline & \% editability & 4.50 & 5.10 & 5.20 & 4.80 \\
\hline & number of guides per mutation & 1.98 & 1.63 & 1.73 & 1.56 \\
\hline \multirow[t]{4}{*}{ Mus musculus } & Total number of mutations edited & 81.00 & 46.00 & 74.00 & 47.00 \\
\hline & Total number of guides designed & 147.00 & 93.00 & 133.00 & 83.00 \\
\hline & \% editability & 8.10 & 4.60 & 7.40 & 4.70 \\
\hline & number of guides per mutation & 1.81 & 2.02 & 1.80 & 1.77 \\
\hline \multirow[t]{4}{*}{ Pan paniscus } & Total number of mutations edited & 73.00 & 57.00 & 62.00 & 54.00 \\
\hline & Total number of guides designed & 123.00 & 89.00 & 98.00 & 90.00 \\
\hline & \% editability & 7.30 & 5.70 & 6.20 & 5.40 \\
\hline & number of guides per mutation & 1.68 & 1.56 & 1.58 & 1.67 \\
\hline \multirow{2}{*}{$\begin{array}{l}\text { Saccharomyces } \\
\text { cerevisiae }\end{array}$} & Total number of mutations edited & 36.00 & 30.00 & 36.00 & 35.00 \\
\hline & Total number of guides designed & 52.00 & 45.00 & 46.00 & 52.00 \\
\hline
\end{tabular}


bioRxiv preprint doi: $\mathrm{https} / /$ doi org/10.1101/426973; this version posted March 8, 2019. The copyright holder for this preprint (which was not certified by peer review) is the author/funder, who has granted bioRxiv a license to display the preprint in perpetuity. It is made available under aCC-BY-NC-ND 4.0 International license.

\begin{tabular}{|l|l|r|r|r|r|}
\hline \multirow{3}{*}{ Sus scrofa } & \% editability & 3.60 & 3.00 & 3.60 & 3.50 \\
\cline { 2 - 5 } & number of guides per mutation & 1.44 & 1.50 & 1.28 & 1.49 \\
\hline & Total number of mutations edited & 68.00 & 50.00 & 55.00 & 45.00 \\
\cline { 2 - 6 } & Total number of guides designed & 108.00 & 106.00 & 86.00 & 82.00 \\
\cline { 2 - 6 } & \% editability & 6.80 & 5.00 & 5.50 & 4.50 \\
\cline { 2 - 6 } & number of guides per mutation & 1.59 & 2.12 & 1.56 & 1.82 \\
\hline
\end{tabular}


Table S7: Summary statistics for case study analysis of clinically relevant human SNPs.

\begin{tabular}{|l|r|r|r|r|}
\hline Mutation format & nucleotide & \multicolumn{1}{l|}{$\begin{array}{l}\text { amino } \\
\text { acid }\end{array}$} & nucleotide & $\begin{array}{l}\text { amino } \\
\text { acid }\end{array}$ \\
\hline Remove/create mutation & remove & remove & create & create \\
\hline $\begin{array}{l}\text { Total number of mutations in the } \\
\text { input data }\end{array}$ & 61083 & 81819 & 61083 & 81819 \\
\hline Total number of mutations edited & 13709 & 19996 & 13867 & 20420 \\
\hline Total number of guides designed & 23432 & 35390 & 22587 & 33311 \\
\hline \% editability & 22.44 & 24.43 & 22.70 & 24.95 \\
\hline
\end{tabular}


Table S8: Default pairs of base editors and PAM recognition sequences supported by beditor.

\begin{tabular}{|c|c|c|c|c|c|c|c|c|}
\hline Base editor & \begin{tabular}{|l} 
nucleotide \\
wt
\end{tabular} & $\begin{array}{l}\text { nucleotide } \\
\text { mutation }\end{array}$ & $\begin{array}{l}\text { window } \\
\text { start }\end{array}$ & $\begin{array}{l}\text { window } \\
\text { end }\end{array}$ & \begin{tabular}{|l|} 
guide \\
length
\end{tabular} & PAM & $\begin{array}{l}\text { PAM } \\
\text { position } \\
\end{array}$ & reference \\
\hline BE1 & C & $T$ & 4 & 8 & 20 & NGG & downstream & [17] \\
\hline BE2 & C & $\mathrm{T}$ & 4 & 8 & 20 & NGG & downstream & [17] \\
\hline BE3 & C & $\mathrm{T}$ & 4 & 8 & 20 & NGG & downstream & {$[17]$} \\
\hline HF-BE3 & C & $\mathrm{T}$ & 4 & 8 & 20 & NGG & downstream & [17] \\
\hline BE4/BE4max & C & $\mathrm{T}$ & 4 & 8 & 20 & NGG & downstream & {$[18,19]$} \\
\hline BE4-Gam & C & $\mathrm{T}$ & 4 & 8 & 20 & NGG & downstream & [18] \\
\hline YE1-BE3 & C & $\mathrm{T}$ & 5 & 7 & 20 & NGG & downstream & [20] \\
\hline EE-BE3 & C & $\mathrm{T}$ & 5 & 6 & 20 & NGG & downstream & [20] \\
\hline YE2-BE3 & C & $\mathrm{T}$ & 5 & 6 & 20 & NGG & downstream & [20] \\
\hline YEE-BE3 & C & $\mathrm{T}$ & 5 & 6 & 20 & NGG & downstream & [20] \\
\hline VQR-BE3 & C & $\mathrm{T}$ & 4 & 11 & 20 & NGAN & downstream & [20] \\
\hline VRER-BE3 & C & $\mathrm{T}$ & 3 & 10 & 20 & NGCG & downstream & [20] \\
\hline SaBE3 & C & $\mathrm{T}$ & 3 & 12 & 21 & NNGRRT & downstream & [20] \\
\hline SaBE4 & C & $\mathrm{T}$ & 3 & 12 & 21 & NNGRRT & downstream & [18] \\
\hline SaBE4-Gam & C & $\mathrm{T}$ & 3 & 12 & 21 & NNGRRT & downstream & [18] \\
\hline SA(KKH)-BE3 & C & $\mathrm{T}$ & 3 & 12 & 21 & NNNRRT & downstream & [20] \\
\hline Cas12a-BE & C & $\mathrm{T}$ & 10 & 12 & 23 & TTTV & upstream & [21] \\
\hline Target-AID & C & $\mathrm{T}$ & 2 & 4 & 20 & NGG & downstream & [22] \\
\hline Target-AID & C & $\mathrm{T}$ & 2 & 4 & 20 & NG & downstream & [23] \\
\hline XBE3 & C & $\mathrm{T}$ & 4 & 8 & 20 & $N G$ & downstream & [6] \\
\hline eA3A-BE3 & C & $\mathrm{T}$ & 4 & 8 & 20 & NGG & downstream & [24] \\
\hline A3A-BE3 & C & $\mathrm{T}$ & 4 & 8 & 20 & NGG & downstream & [25] \\
\hline BE-PLUS & C & $\mathrm{T}$ & 4 & 14 & 20 & NGG & downstream & [26] \\
\hline ABE7.9 & A & $G$ & 5 & 8 & 20 & NGG & downstream & [17] \\
\hline ABE7.10 & A & $G$ & 4 & 7 & 20 & NGG & downstream & {$[17]$} \\
\hline ABE7.10* & A & $G$ & 4 & 8 & 20 & NGG & downstream & [17] \\
\hline XABE7.10 & A & $G$ & 4 & 7 & 20 & NG & downstream & [6] \\
\hline ABESa & A & $G$ & 6 & 12 & 21 & NNGRRT & downstream & {$[6,27]$} \\
\hline VQR-ABE & A & $G$ & 4 & 6 & 20 & NGA & downstream & {$[6,27]$} \\
\hline VRER-ABE & $A$ & $G$ & 4 & 6 & 20 & NGCG & downstream & {$[6,27]$} \\
\hline
\end{tabular}


bioRxiv preprint doi: https://doi.org/10.1101/426973; this version posted March 8, 2019. The copyright holder for this preprint (which was not certified by peer review) is the author/funder, who has granted bioRxiv a license to display the preprint in perpetuity. It is made available under aCC-BY-NC-ND 4.0 International license.

\begin{tabular}{|l|l|l|r|r|r|l|l|l|}
\hline $\mathrm{Sa}(\mathrm{KKH})-\mathrm{ABE}$ & A & G & 6 & 12 & 21 & NNNRRT & downstream & {$[6,27]$} \\
\hline
\end{tabular}




\section{References}

1. Billon P, Bryant EE, Joseph SA, Nambiar TS, Hayward SB, Rothstein R, et al. CRISPRMediated Base Editing Enables Efficient Disruption of Eukaryotic Genes through Induction of STOP Codons. Mol Cell [Internet]. Elsevier Inc.; 2017;67:1068-1079.e4. Available from: http://dx.doi.org/10.1016/j.molcel.2017.08.008

2. Hwang G, Park J, Lim K, Kim S, Yu J, Eils R, et al. Web-based design and analysis tools for CRISPR base editing. bioRxiv [Internet]. 2018;5:373944. Available from: https://www.biorxiv.org/content/early/2018/07/22/373944?rss=1\&utm_source=dlvr.it\&ut m_medium=twitter

3. Benchling Inc. Benchling for Academics · Benchling. Benchling. 2018.

4. Komor AC, Kim YB, Packer MS, Zuris JA, Liu DR. Programmable editing of a target base in genomic DNA without double-stranded DNA cleavage. Nature. Nature Publishing Group; 2016;533:420.

5. Doench JG, Fusi N, Sullender M, Hegde M, Vaimberg EW, Donovan KF, et al. Optimized sgRNA design to maximize activity and minimize off-target effects of CRISPRCas9. Nat Biotechnol [Internet]. Nature Publishing Group; 2016;34:184-91. Available from: http://dx.doi.org/10.1038/nbt.3437

6. Hu JH, Miller SM, Geurts MH, Tang W, Chen L, Sun N, et al. Evolved Cas9 variants with broad PAM compatibility and high DNA specificity. Nature [Internet]. Nature Publishing Group; 2018;556:57-63. Available from: http://dx.doi.org/10.1038/nature26155

7. Kleinstiver BP, Prew MS, Tsai SQ, Topkar V V., Nguyen NT, Zheng Z, et al. Engineered CRISPR-Cas9 nucleases with altered PAM specificities. Nature. 2015;

8. Hsu PD, Scott DA, Weinstein JA, Ran FA, Konermann S, Agarwala V, et al. DNA targeting specificity of RNA-guided Cas9 nucleases. Nat Biotechnol. 2013;31:827-32.

9. Jinek M, Chylinski K, Fonfara I, Hauer M, Doudna JA, Charpentier E. A programmable dual-RNA-guided DNA endonuclease in adaptive bacterial immunity. Science (80- ). 2012;

10. Magadán AH, Dupuis MÈ, Villion M, Moineau S. Cleavage of phage DNA by the Streptococcus thermophilus CRISPR3-Cas system. PLoS One. 2012;

11. Garneau JE, Dupuis MÈ, Villion M, Romero DA, Barrangou R, Boyaval P, et al. The CRISPR/cas bacterial immune system cleaves bacteriophage and plasmid DNA. Nature. 2010;

12. Kleinstiver BP, Prew MS, Tsai SQ, Nguyen NT, Topkar V V., Zheng Z, et al. Broadening the targeting range of Staphylococcus aureus CRISPR-Cas9 by modifying PAM recognition. Nat Biotechnol. 2015;

13. Gasiunas G, Barrangou R, Horvath P, Siksnys V. Cas9-crRNA ribonucleoprotein 
complex mediates specific DNA cleavage for adaptive immunity in bacteria. Proc Natl Acad Sci U S A. 2012;

14. Hou Z, Zhang Y, Propson NE, Howden SE, Chu L, Sontheimer EJ, et al. Efficient genome engineering in human pluripotent stem cells using Cas9 from Neisseria meningitidis. Proc Natl Acad Sci USA. 2013;

15. Gao L, Cox DBT, Yan WX, Manteiga JC, Schneider MW, Yamano T, et al. Engineered Cpf1 variants with altered PAM specificities. Nat Biotechnol [Internet]. Nature Publishing Group; 2017;35:789-92. Available from: http://dx.doi.org/10.1038/nbt.3900

16. Zetsche B, Gootenberg JS, Abudayyeh OO, Slaymaker IM, Makarova KS, Essletzbichler P, et al. Cpf1 Is a Single RNA-Guided Endonuclease of a Class 2 CRISPRCas System. Cell. 2015;

17. Gaudelli NM, Komor AC, Rees HA, Packer MS, Badran AH, Bryson DI, et al. Programmable base editing of $A \cdot T$ to $G \cdot C$ in genomic DNA without DNA cleavage. Nature [Internet]. Nature Publishing Group; 2017;551:464-71. Available from: http://www.nature.com/doifinder/10.1038/nature24644

18. Komor AC, Zhao KT, Packer MS, Gaudelli NM, Waterbury AL, Koblan LW, et al. Improved base excision repair inhibition and bacteriophage Mu Gam protein yields C:Gto-T:A base editors with higher efficiency and product purity. Sci Adv. 2017;

19. Koblan LW, Doman JL, Wilson C, Levy JM, Tay T, Newby GA, et al. Improving cytidine and adenine base editors by expression optimization and ancestral reconstruction. Nat Biotechnol. 2018;

20. Kim YB, Komor AC, Levy JM, Packer MS, Zhao KT, Liu DR. Increasing the genometargeting scope and precision of base editing with engineered Cas9-cytidine deaminase fusions. Nat Biotechnol [Internet]. Nature Publishing Group; 2017;35:371-6. Available from: http://dx.doi.org/10.1038/nbt.3803

21. Li X, Wang Y, Liu Y, Yang B, Wang X, Wei J, et al. Base editing with a Cpf1 - cytidine deaminase fusion. 2018;36.

22. Nishida K, Arazoe T, Yachie N, Banno S, Kakimoto M, Tabata M, et al. Targeted nucleotide editing using hybrid prokaryotic and vertebrate adaptive immune systems. 2016;8729:1-14.

23. Nishimasu H, Shi X, Ishiguro S, Gao L, Hirano S, Okazaki S, et al. Engineered CRISPR-Cas9 nuclease with expanded targeting space. Science (80- ) [Internet]. 2018;9129:eaas9129. Available from: http://www.sciencemag.org/lookup/doi/10.1126/science.aas9129

24. Gehrke JM, Cervantes O, Clement MK, Wu Y, Zeng J, Bauer DE, et al. An APOBEC3A-Cas9 base editor with minimized bystander and off-target activities. Nat Biotechnol [Internet]. Nature Publishing Group; 2018; Available from: http://dx.doi.org/10.1038/nbt.4199 
25. Gehrke JM, Cervantes OR, Clement MK, Pinello L, Joung JK, Unit MP, et al. Highprecision CRISPR-Cas9 base editors with minimized bystander and off-target mutations. bioRxiv [Internet]. 2018;273938. Available from: https://www.biorxiv.org/content/early/2018/03/01/273938

26. Jiang W, Feng S, Huang S, Yu W, Li G, Yang G, et al. BE-PLUS: a new base editing tool with broadened editing window and enhanced fidelity. Cell Res. 2018;

27. Hua K, Tao X, Zhu JK. Expanding the base editing scope in rice by using Cas9 variants. Plant Biotechnol J. 2019; 